\title{
$T^{3}$-ACTIONS ON SIMPLY CONNECTED 6-MANIFOLDS.I
}

BY

\section{DENNIS McGAVRAN}

\begin{abstract}
We are concerned with $T^{3}$-actions on simply connected 6-manifolds $M^{6}$. As in the codimension two case, there exists, under certain restrictions, a cross-section. Unlike the codimension two case, the orbit space need not be a disk and there can be finite stability groups. C. T. C. Wall has determined (Invent. Math. 1 (1966), 355-374) a complete set of invariants for simply connected 6-manifolds with $H_{*}\left(M^{6}\right)$ torsion-free and $\omega_{2}\left(M^{6}\right)$ $=0$. We establish sufficient conditions for these two requirements to be met when $M$ is a $T^{3}$-manifold. Using surgery and connected sums, we compute the invariants for manifolds satisfying these conditions. We then construct a $T^{3}$-manifold $M^{6}$ with invariants different than any well-known manifold. This involves comparing the trilinear forms (defined by Wall) for two different manifolds.
\end{abstract}

There has been much work done recently on the classification of torus actions on manifolds. Complete results have been obtained in certain cases: $S^{\mathbf{1}}$. actions on closed, compact, connected 3-manifolds ([14], [17]), and $T^{2}$-actions on closed, compact, connected, simply connected 4-manifolds [15]. In the more general setting, Pak has shown in [16] that, for $n \geqslant 3, T^{n+1}$ and $L(k, q) \times$ $T^{n-2}$ are the only compact, closed, orientable, $(n+1)$-manifolds admitting a $T^{n}$-action. Various results are obtained in [8] concerning actions of $T^{n}$ on closed, compact, simply connected manifolds of codimensions two and four. In particular, examples of effective actions are given in each case for all $n \geqslant 1$.

In this paper we begin the study of the codimension three case by looking at $T^{n}$-actions on closed, compact, connected, simply connected 6-manifolds. This case is also similar to [15] in that the dimension of the manifold is twice the dimension of the torus. One might expect things to become a little more complicated in this case and this is, in fact, true. For $T^{n}$-actions on simply connected $(n+2)$-manifolds, the orbit space is a disk, $D^{2}$, and there are no finite stability groups. We provide examples in $\S 3$ to show that, for $T^{3}$-actions on

Presented to the Society, April 13, 1974; received by the editors August 7, 1974 and, in revised form, March 10, 1975.

AMS (MOS) subject classifications (1970). Primary 57E25, 57E10, 57E15; Secondary 57D20, 57D65.

Key words and phrases. Group actions, 6-manifold, torus, orbit space, cross-section, second Stiefel-Whitney class, surgery, connected sums, trilinear forms.

Copyright $\bigcirc$ 1976. Amurican Mathernatical So:icty 
simply connected 6-manifolds, the orbit space may be $S^{2} \times I$, there may be finite stability groups, and there may or may not be fixed points. For most of our work we assume that the orbit space is $D^{3}$, that there are fixed points, and that there are no finite stability groups.

In $\S 4$ we prove results about the orbit structure and we also examine the relationship between the stability group structure and the second Stiefel-Whitney class $\omega_{2}\left(M^{6}\right)$. $\S 5$ describes a particular $T^{3}$-action. In $\S 6$ we prove a cross-sectioning theorem and an equivariant classification theorem. The techniques we use are similar to those used in [15].

In $\S 7$ we concern ourselves with identifying which simply connected 6-manifolds admit $T^{3}$-actions. Our main tool is a result of C. T. C. Wall (see [20]). Roughly speaking, Wall shows that a simply connected 6-manifold, $M$, with $\omega_{2}(M)=0$ and $H_{*}(M)$ torsion-free, is determined, up to diffeomorphism, by its cohomology ring $H^{*}(M)$ and its first Pontrjagin class, $p_{1}(M)$. If $M$ admits a $T^{3}$. action, we obtain certain conditions which must be satisfied by these invariants and give a partial description of what $M$ must be. Considering the work done by Orlik and Raymond (see [15]), one might expect, under the conditions we impose, that $M$ would be a connected sum of copies of $S^{6}, S^{3} \times S^{3}, S^{2} \times S^{4}$, $C P(3)$, and manifolds of the form $S^{2} \times\left(S^{2} \times S^{2} \# \cdots \# S^{2} \times S^{2}\right)$. In $\S 8$, we provide an example to show that this is not the case.

In a future paper we intend to investigate the possibilities when there are no fixed points and when $M^{*}$ is a connected sum of copies of $S^{2} \times I$.

We assume that all manifolds under consideration are closed, compact, connected and orientable. Also all actions are assumed to be locally smooth and effective. We let $\mathbf{Z}, \mathbf{Q}, \mathbf{R}$ denote the integers, rational numbers and real numbers, respectively. We use $\stackrel{\bigcirc}{X}$ to denote the interior of the space $X$. Unless otherwise indicated, the coefficients in all (co)homology groups will be $Z$. We use $I$ to indicate the unit interval $[0,1]$, while $S^{n-1}$ and $D^{n-1}$ indicate the unit sphere and unit disk in $\mathrm{R}^{n}, n>1 . X \cong_{\mathrm{eq}} Y$ will mean that the $G$-spaces, $X$ and $Y$, are equivariantly homeomorphic. The reader may refer to [1] for standard definitions relating to transformation groups.

This paper constitutes part of the author's Ph. D. dissertation at Wayne State University, written under Professor Jingyal Pak. Many missing details can be found there.

1. Wall's results for simply connected 6-manifolds. We now describe the results of C. T. C. Wall on simply connected 6-manifolds (see [20]). His results hold for manifolds satisfying the hypothesis

(H) $H_{*}(M)$ is torsion-free and $\omega_{2}(M)=0$.

Let $M$ be a closed, smooth, simply connected 6-manifold satisfying (H). We 
first orient $M$, thereby determining a particular isomorphism $H^{6}(M) \rightarrow \mathbf{Z}$. Let $H=H^{2}(M) \cong H_{4}(M)$. Then $\operatorname{Hom}_{\mathrm{Z}}(H, \mathrm{Z}) \cong H^{4}(M) \cong H_{2}(M)$. The isomorphism $H^{6}(M) \rightarrow \mathrm{Z}$ and the cup product determine a trilinear form $\mu: H \times H \times H \rightarrow$ $\mathrm{Z}$ defined by $\mu(\alpha, \beta, \gamma)=a \cup \beta \cup \gamma$. The first Pontrjagin class $p_{1}(M) \in H^{4}(M)$ determines a homomorphism $p_{1}: H \rightarrow \mathrm{Z}$. Let $G=H^{3}(M)$. Wall's major result is the following:

1.1 THEOREM (Theorem 5 of [20]). Diffeomorphism classes of oriented manifolds satisfying $(\mathrm{H})$ correspond bijectively to isomorphism classes of systems of invariants:

two free abelian groups $G$ and $H$

a symmetric trilinear map $\mu: H \times H \times H \rightarrow \mathbf{Z}$

a homomorphism $p_{1}: H \rightarrow \mathrm{Z}$

subject to:

$$
\begin{aligned}
& \text { for } x, y \in H \mu(x, x, y) \equiv \mu(x, y, y)(\bmod 2) \\
& \text { for } x \in H p_{1}(x) \equiv 4 \mu(x, x, x)(\bmod 24) .
\end{aligned}
$$

Wall shows how these invariants can be computed and we now examine these computations.

Suppose that $M$ is a closed, simply connected, oriented, 6-manifold satisfying (H). Wall first shows that $M \cong M_{1} \# M_{2}$, where $M_{2}$ is a connected sum of $k$ copies of $S^{3} \times S^{3}, 2 k=\mathrm{rk} H^{3}(M)$. Hence, we may assume $H^{3}(M)=0$. In this case, Wall proves the following.

1.2 Theorem. Suppose $M$ satisfies $(H)$ and $H^{3}(M)=0$. Then $M$ can be obtained from $S^{6}$ by surgery on a disjoint set of embeddings $\bar{g}_{i}: S^{3} \times D^{3} \rightarrow S^{6}$.

Given an embedding $\bar{g}_{i}$, we obtain $g_{i}: S^{3} \cong\left(S^{3} \times 0\right) \rightarrow S^{6}$. Let $\left(S^{3} \times D^{3}\right)_{i}$ and $S_{i}^{3}$ denote the images of $\bar{g}_{i}$ and $g_{i}$, respectively. When we do surgery, we replace $\left(S^{3} \times D^{3}\right)_{i}$ with $\left(S^{2} \times D^{4}\right)_{i}$. Then $S_{i}^{2}=\left(S^{2} \times 0\right)_{i}$ corresponds to a basis element $e_{i}$ of $H^{2}(M)$. Now choose an $S^{2}$ embedded in $S^{6}$ such that its linking number with $S_{j}^{3}$ is +1 (to fix signs) and a homotopy equivalence between $S^{6}-S_{j}^{3}$ and this $S^{2}$. We then obtain an element of $\pi_{3}\left(S^{2}\right)$, represented by the composition of $g_{i}$ with this homotopy equivalence. Choose the Hopf map as a generator of $\pi_{3}\left(S^{2}\right)$. Sending this to $+1 \in \mathrm{Z}$ determines an isomorphism $\pi_{3}\left(S^{2}\right)$ $\rightarrow Z$. Our above composition then determines an integer $\lambda_{j}^{l}$.

Choose the identity map as a generator for $\pi_{3}\left(S^{3}\right)$, determining, as above, an isomorphism $\pi_{3}\left(S^{3}\right) \rightarrow Z$. We have an isomorphism

$$
\pi_{3}\left(S^{2} \vee S^{2}\right)=\pi_{3}\left(S^{2}\right) \oplus \pi_{3}\left(S^{2}\right) \oplus \pi_{3}\left(S^{3}\right)
$$

where the third summand is injected by the Whitehead product of the inclusion maps of the two copies of $S^{2}$. Choosing a homotopy equivalence between $S^{6}-$ 
$\left(S_{j}^{3} \cup S_{k}^{3}\right)$ and $S^{2} \vee S^{2}$, and composing this with $g_{i}$, we get an element of $\pi_{3}\left(S^{2} \bigvee S^{2}\right)$. Projecting this onto $\pi_{3}\left(S^{3}\right)$, we obtain an integer $\lambda_{j k}^{i}$.

Let $F C_{3}^{3}$ be the group of isotopy classes of embeddings $g: S^{3} \times D^{3} \rightarrow S^{6}$, and $C_{3}^{3}$ the group of isotopy classes of embeddings $\bar{g}: S^{3} \rightarrow S^{6}$. By taking as a generator for $C_{3}^{3}$ the embedding given in [5], we obtain an isomorphism $C_{3}^{3} \rightarrow \mathbf{Z}$. Also, choose a particular isomorphism $\pi_{3}(S O(3)) \rightarrow Z$. We have the following exact sequence:

$$
0 \rightarrow \pi_{3}(S O(3)) \stackrel{\tau}{\rightarrow} F C_{3}^{3} \stackrel{\varphi}{\rightarrow} C_{3}^{3} \rightarrow 0
$$

where $\tau$ twists the tubular neighborhood $g$ of an embedding $\bar{g}: S^{3} \rightarrow S^{6}$. Since $\pi_{3}(S O(3))$ and $C_{3}^{3}$ are both infinite cyclic, the sequence splits and we have a splitting map $\beta^{\prime}: F C^{3} \rightarrow \pi_{3}(S O(3)$ ). (An explicit map is chosen in [20].) Then, if $g_{i}: S^{3} \times D^{3} \rightarrow S^{6}$ is an embedding, $\varphi\left(g_{i}\right)$ and $\beta^{\prime}\left(g_{i}\right)$ determine integers $\varphi_{i}$ and $\beta_{i}^{\prime}$. Wall proves the following:

\subsection{Theorem. Given $M$ as in 1.1}

$$
\begin{aligned}
& \mu\left(e_{i}, e_{j}, e_{k}\right)=\lambda_{j k}^{i}, \quad p_{1}\left(e_{i}\right)=4 \beta_{i}^{\prime}, \\
& \mu\left(e_{j}, e_{j}, e_{i}\right)=\lambda_{j}^{i}, \\
& \mu\left(e_{i}, e_{i}, e_{i}\right)=6 \varphi_{i}+\beta_{i}^{\prime} .
\end{aligned}
$$

We shall need explicitly the homotopy equivalences mentioned above. Hence, let

$$
\begin{aligned}
S^{6}= & \left\{z=\left(r_{1} e^{i \theta_{1}}, r_{2} e^{i \theta_{2}}, r_{3} e^{i \theta_{3}}, r_{4}\right) \mid \sum r_{i}^{2}=1 ; r_{i} \geqslant 0 \text { for } i \neq 4\right\}, \\
& D_{+}^{6}=\left\{z \in S^{6} \mid r_{4} \geqslant 0\right\}, \quad D_{-}^{6}=\left\{z \in S^{6} \mid r_{4} \leqslant 0\right\} .
\end{aligned}
$$

For $\alpha=1,2,3$ let

$$
\begin{gathered}
S_{\alpha}^{3}=\left\{z \in S^{6} \mid r_{\alpha}=r_{4}=0\right\}, \quad S_{\alpha}^{2}=\left\{z \in S^{6} \mid r_{k}=0 \text { for } k \neq \alpha, 4\right\}, \\
S_{\alpha \pm}^{3}=\left\{z \in S^{6} \mid r_{\alpha}=0, r_{4}= \pm 1 / 2\right\} .
\end{gathered}
$$

Now $S^{6}-S_{\alpha}^{3}$ is homotopy equivalent to $S^{6}-\left(S_{\alpha}^{3} \times D^{3}\right)$, and $S_{\alpha}^{2}$ is a 2-chain representing a generator of $H_{2}\left(S^{6}-\left(S_{\alpha}^{3} \times D^{3}\right)\right)$. We assume, for convenieince, that orientations are chosen so that $S_{\alpha}^{3}$ and $S_{\alpha}^{2}$ have linking number +1 .

Let $f:\left(S^{6}-S_{1}^{3}\right) \rightarrow S_{1}^{2}$ be defined by

$$
f(z)=\left(\frac{r_{1}}{\sqrt{R_{1}}} e^{i \theta_{1}}, 0,0, \frac{r_{4}}{\sqrt{R_{1}}}\right), \quad R_{1}=r_{1}^{2}+r_{4}^{2} .
$$

It is shown in [11] that this is a homotopy equivalence. Obvious modifications will give the desired maps between $S^{6}-S_{\alpha}^{3}$ and $S_{\alpha^{\prime}}^{2}, \alpha=2,3$.

Now let $S_{+}^{6}$ and $S_{-}^{6}$ be two copies of $S^{6}$. Define $h_{+}: D_{+}^{6} \rightarrow S_{+}^{6}$ and $h_{-}$: $D_{-}^{6} \rightarrow S_{-}^{6}$ as follows: 


$$
h_{ \pm}(z)=\left(2 r_{1} \sqrt{\frac{r_{4}}{r_{4} \pm 1}} e^{i \theta_{1}}, 2 r_{2} \sqrt{\frac{r_{4}}{r_{4} \pm 1}} e^{i \theta_{2}}, 2 r_{3} \sqrt{\frac{r_{4}}{r_{4} \pm 1}} e^{i \theta_{3}}, 2 r_{4} \mp 1\right) \text {. }
$$

We then define

$$
h=h_{+} \vee h_{-}:\left(S^{6}-\left(S_{\beta+}^{3} \cup S_{\alpha-}^{3}\right)\right) \rightarrow\left(S_{+}^{6}-S_{\beta}^{3}\right) \vee\left(S_{-}^{6}-S_{\alpha}^{3}\right)
$$

By our previous work, we have a homotopy equivalence between $\left(S_{+}^{6}-S_{\beta}^{3}\right) \vee$ $\left(S_{-}^{6}-S_{\alpha}^{3}\right)$ and $S_{\beta}^{2} \vee S_{\alpha}^{2}$. In [11] it is shown that $h$ is a homotopy equivalence so the composition gives a homotopy equivalence between $S^{6}-\left(S_{\beta+}^{3} \cup S_{\alpha-}^{3}\right)$ and $S_{\beta}^{2} \vee S_{\alpha}^{2}$.

2. Trilinear forms. Suppose $V$ is a $Z$-module. A trilinear form on $V$ is a function $\varphi: V \times V \times V \rightarrow \mathbf{Z}$ that is linear in each factor. Suppose $B=$ $\left\{e_{1}, \ldots, e_{n}\right\}$ is a basis for $V$. We then obtain a three dimensional matrix representation $M=(\varphi)_{B}$ for $\varphi$ with respect to $B$ by letting $M=\left(m_{i j k}\right), m_{i j k}=$ $\varphi\left(e_{i}, e_{j}, e_{k}\right)$. More generally, if we choose bases $B_{1}, B_{2}$, and $B_{3}$ for the three factors of $V$, we obtain a matrix $(\varphi)_{B_{1} B_{2} B_{3}}$ in the obvious manner. We picture $M$ as follows:

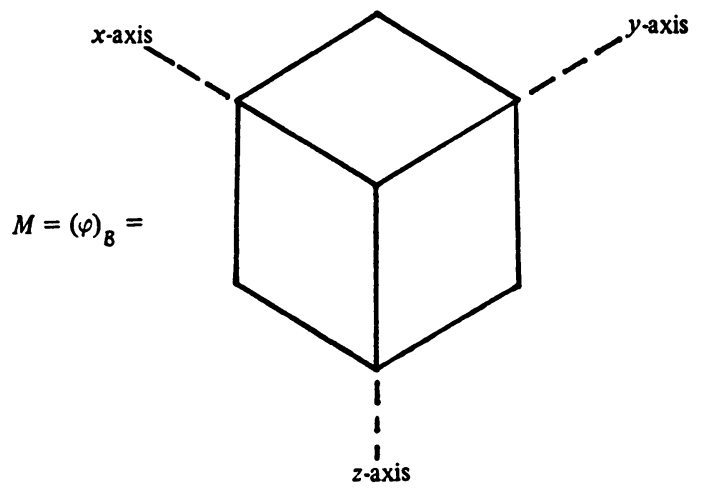

where the three axes correspond to the respective factors of $V$. We have the following interpretation of $\left.M . \varphi\left(e_{j},{ }_{-},\right)\right)$is a bilinear form on $V$. Its matrix with respect to $B,\left(\varphi\left(e_{j},-,-\right)\right)_{B}$, is the slice through $M$ perpendicular to the $x$-axis, corresponding to the basis element $e_{j}$. Similarly, the matrices for $\left.\varphi_{-}, e_{j},-\right)$ and $\varphi\left(,,, e_{j}\right)$ are slices through $M$ perpendicular to the $y$ - and $z$-axes, respectively.

Now suppose we have bases $B=\left\{e_{i}\right\}$ and $B^{\prime}=\left\{e_{i}^{\prime}\right\}$ for $V$. Let $A=\left(a_{i j}\right)$ be the change of basis matrix, i.e., $e_{j}=\Sigma_{i=1}^{n} a_{i j} e_{i}^{\prime}$. Let $M=(\varphi)_{B}$ and $M^{\prime}=(\varphi)_{B^{\prime}}$. We wish to show how $M$ and $M^{\prime}$ are related.

We first show how the matrix $(\varphi)_{B B^{\prime} B^{\prime}}$ is related to $M$. A slice through $M$ perpendicular to the $x$-axis is the matrix $\left(\varphi\left(e_{j}, \ldots,-\right)\right)_{B}$, while a slice through $(\varphi)_{B B^{\prime} B^{\prime}}$ is the matrix $\left(\varphi\left(e_{j},-,-\right)\right)_{B^{\prime}}$. Hence, the following relations hold between $M$ and $(\varphi)_{B B^{\prime} B^{\prime}}$ : 


$$
A\left(\varphi\left(e_{j}, \ldots,-\right)\right)_{B} A^{T}=\left(\varphi\left(e_{j}, \ldots,-\right)\right)_{B^{\prime \prime}}
$$

Now the slices through $(\varphi)_{B_{B^{\prime}} B^{\prime}}$ perpendicular to the $y$-axis are the matrices $\left(\varphi\left(-, e_{j}^{\prime},-\right)\right)_{B B^{\prime}}$, the matrices for the bilinear forms $\left.\varphi_{-}, e_{j}^{\prime},-\right)$ with respect to the bases $B$ and $B^{\prime}$. The slices perpendicular to the $y$-axis in $M^{\prime}$ are the matrices $\left(\varphi\left(-, e_{j}^{\prime},-\right)\right)_{B^{\prime}}$. Hence, we have the following relations:

$$
A\left(\varphi\left(\_, e_{j}^{\prime}, \_\right)\right)_{B B^{\prime}}=\left(\varphi\left(C_{-}, e_{j}^{\prime},\right)_{B^{\prime}}\right.
$$

Schematically we have
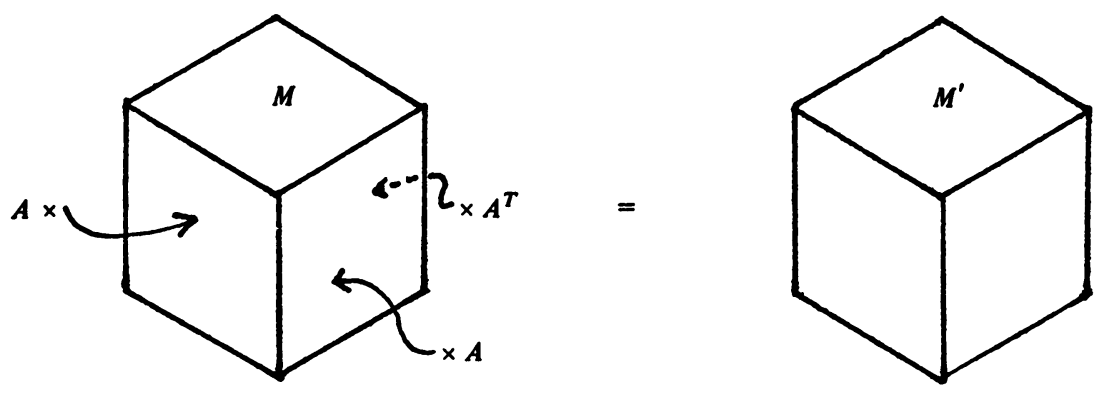

or more compactly,

$$
A M A^{T}=M^{\prime}
$$

Equivalently, if $B=A^{-1}$, we have

$$
A M A^{T}=\stackrel{M^{\prime}}{B} .
$$

3. Examples. Let $T^{3}=\left\{t=\left(e^{i \varphi_{1}}, e^{i \varphi_{2}}, e^{i \varphi_{3}}\right) \mid 0 \leqslant \varphi_{i}<2 \pi\right\}=T_{1} \times T_{2}$ $\times T_{3}$. We give some examples of $T^{3}$-spaces.

3.1. The obvious action of $T^{3}$ on $S^{6}$ has the weighted orbit space (indicating the isotropy type of the orbits) shown below.

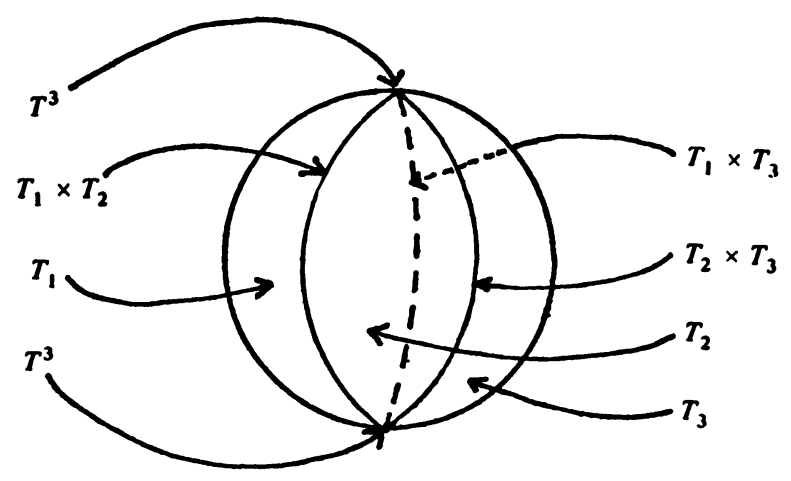

(The interior points are principal orbits.)

Note that $D^{6}=\left\{z \in S^{6} \mid r_{4} \geqslant 0\right\}$. By restriction, we have a $T^{3}$-action on 
$D^{6}$ with orbit space the upper half of $D^{3}$. Furthermore, $S^{5}=\left\{z \in S^{6} \mid r_{4}=0\right\}$ and we get an action $\left(T^{3}, S^{5}\right)$ with orbit space $S^{5} / T^{3} \cong D^{2}$. (Slice a plane through $\left(S^{6}\right)^{*}$ at the equator.)

3.2. Let $S^{7}=\left\{z=\left(r_{1} e^{i \theta_{1}}, r_{2} e^{i \theta_{2}}, r_{3} e^{i \theta_{3}}, r_{4} e^{i \theta_{4}}\right) \mid \Sigma r_{i}^{2}=1 ; r_{i} \geqslant 0\right\}$. We have the natural action of $T^{1}$ on $S^{7}$ such that $S^{7} / T^{1}=C P(3)$. Let $\bar{z}$ denote the equivalence class in $C P(3)$ of $z \in S^{7}$. For $t \in T^{3}$, define

$$
t \bar{z}=\overline{\left(r_{1} e^{i\left(\theta_{1}+\varphi_{1}\right)}, \ldots, r_{3} e^{i\left(\theta_{3}+\varphi_{3}\right)}, r_{4} e^{i \theta_{4}}\right)}
$$

Again, we have the orbit space $C P(3) / T^{3} \cong D^{3}$, as shown below:

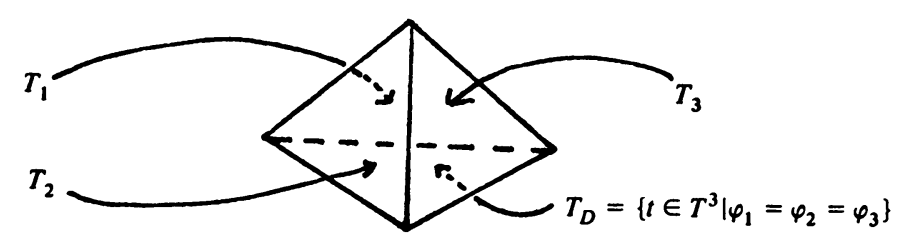

The vertices are fixed points, the edges correspond to the appropriate $T^{2}$-subgroups, and the interior points are principal orbits. It is easy to see that each circle stability group fixes a copy of $C P(2)$.

3.3. We have the following general example. Suppose we have an action of $T_{1} \times T_{2}$ on a simply connected 4-manifold $M$. We know by [15] that $M$ is a connected sum of $C P(2)$ 's, $S^{2} \times S^{2}$ 's, and $S^{4}$ 's. Taking the product of this action with the natural action $\left(T_{3}, S^{2}\right)$, we get an action $\left(T^{3}, M \times S^{2}\right)$. We have $F\left(T^{3}, M \times S^{2}\right)=\left\{(p,(0, \pm 1)) \mid p \in F\left(T_{1} \times T_{2}, M\right)\right\}$. Hence, if $\left(T_{1} \times T_{2}, M\right)$ has $n$ fixed points, $\left(T^{3}, M \times S^{2}\right)$ has $2 n$ fixed points. Also, $F\left(T_{3}, M \times S^{2}\right)=M$ $\times S^{0}$, and, if $T$ is a circle stability group of $\left(T_{1} \times T_{2}, M\right)$, then $F\left(T, M \times S^{2}\right)=$ $F(T, M) \times S^{2}$. We can see that the orbit space is of the form

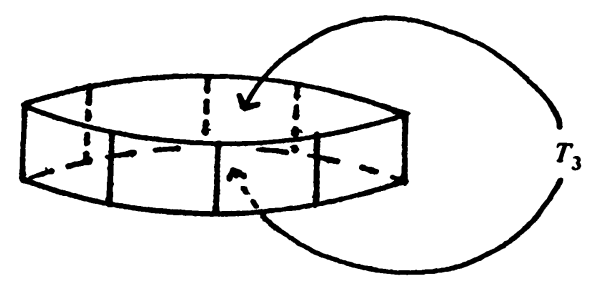

Specifically we obtain actions $\left(T^{3}, S^{2} \times S^{4}\right)$ with four fixed points, $\left(T^{3}, S^{2} \times\right.$ $\left.S^{2} \times S^{2}\right)$ with eight fixed points, and $\left(T^{3}, S^{2} \times C P(2)\right)$ with six fixed points. Note that $\omega_{2}\left(S^{2} \times C P(2)\right) \neq 0$.

We now give some examples of $T^{3}$-actions on $S^{3} \times S^{3}$ which are somewhat more exotic than the previous examples.

3.4. Let $S^{3}=\left\{z=\left(r_{1} e^{i \theta_{1}}, r_{2} e^{i \theta_{2}}\right) \mid r_{1}^{2}+r_{2}^{2}=1\right\}$. Define an action of $T_{1} \times T_{2}$ on $S^{3}$ by $t z=\left(r_{1} e^{i\left(\theta_{1}+\varphi_{1}\right)}, r_{2} e^{i\left(\theta_{2}+\varphi_{2}\right)}\right)$. Then $S^{3} / T_{1} \times T_{2} \cong D^{1}$. Define an action $\left(T_{3}, S^{3}\right)$ by $t z=\left(r_{1} e^{i\left(\theta_{1}+\varphi_{3}\right)}, r_{2} e^{i \theta_{2}}\right)$, so that $S^{3} / T_{3} \cong D^{2}$. 
Hence, we get an action $\left(T^{3}, S^{3} \times S^{3}\right)$ with $S^{3} \times S^{3} / T^{3} \cong D^{3}$. Note that there are no fixed points, and that $F\left(T_{i}, S^{3} \times S^{3}\right)=S^{3} \times S^{1}, i=1,2,3$. The orbit space is:

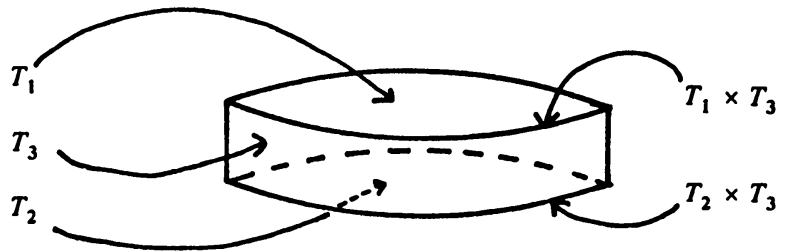

3.5. We have an action of $T_{3}$ on $S^{3}$ defined by the Hopf fibering $S^{3} / T_{3} \cong$ $S^{2}$. If we take the product of this action with the action of $T_{1} \times T_{2}$ on $S^{3}$ defined in 3.4 we get an action $\left(T^{3}, S^{3} \times S^{3}\right)$ with orbit space $S^{2} \times I$. The only stability groups are $T_{1}$ and $T_{2}$ with $F\left(T_{i}, S^{3} \times S^{3}\right) \cong S^{1} \times S^{2}$ for $i=1,2$. In the orbit space, $S^{2} \times 0$ corresponds to one stability group and $S^{2} \times 1$ to the other.

3.6. Now define an action of $T_{3}$ on $S^{3}$ by $t z=\left(r_{1} e^{i\left(\theta_{1}+q \varphi_{3}\right)}, r_{2} e^{i\left(\theta_{2}+q \varphi_{3}\right)}\right)$, $(p, q)=1$. We have stability groups $\mathrm{Z}_{p}$ and $\mathrm{Z}_{q}$ and orbit space $S^{3} / T_{3}=S^{2}$ (see [14]). Again we take the product of this action with the action $\left(T_{1} \times T_{2}\right.$, $\left.S^{3}\right)$ defined in 3.4 to get an action $\left(T^{3}, S^{3} \times S^{3}\right)$. We have stability groups $T_{1}$, $T_{2}, \mathrm{Z}_{p}, \mathrm{Z}_{q}, T_{i} \times \mathrm{Z}_{p}$ and $T_{i} \times \mathrm{Z}_{q}$ for $i=1$, 2. Also, $S^{3} \times S^{3} / T^{3}=S^{2} \times I$.

We now give two methods of obtaining more complicated $T^{3}$-actions from simpler ones.

Suppose we have locally smooth actions $\left(T^{3}, M_{i}\right), i=1,2$, with each $M_{i}^{*}$ $\cong D^{3}$. Suppose we have fixed points $p_{i} \in M_{i}$ such that an invariant neighborhood of each $p_{i}$ is equivariantly homeomorphic to $D^{6}$ with the standard $T^{3}$-action, having stability groups $T_{1}, T_{2}$ and $T_{3}$.
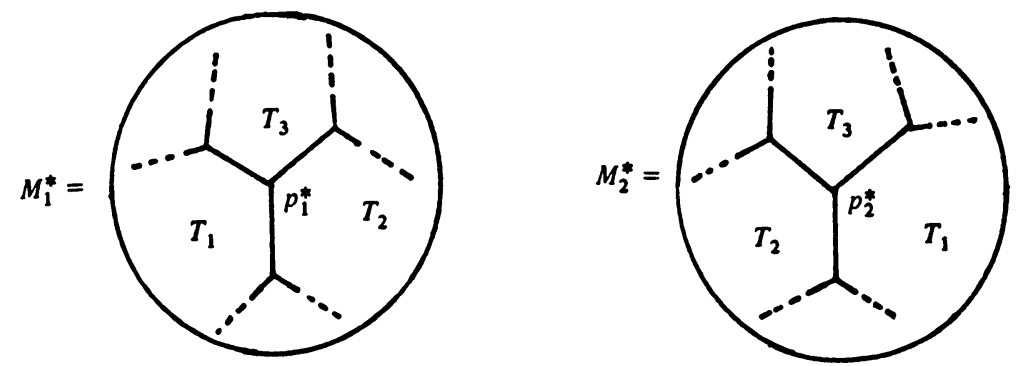

We may then attach $\left(M_{1}-D^{6}\right)$ to $\left(M_{2}-D^{6}\right)$ via an equivariant homeomorphism between their boundaries, which are homeomorphic to $S^{5}$ in each case. We then have an action of $T^{3}$ on $M_{1} \# M_{2}$.

Now let $D^{3}$ have the natural $T_{3}$-action and take the product of this action with the action $\left(T_{1} \times T_{2}, S^{3}\right)$ defined in 3.4. We get a $T^{3}$-action on $S^{3} \times D^{3}$ with orbit space: 


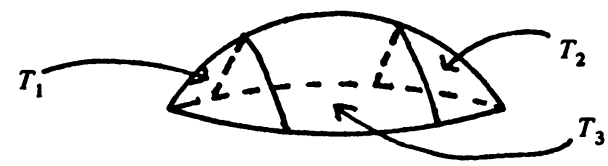

In a similar manner we get a $T^{3}$-action on $S^{2} \times D^{4}$ with orbit space:

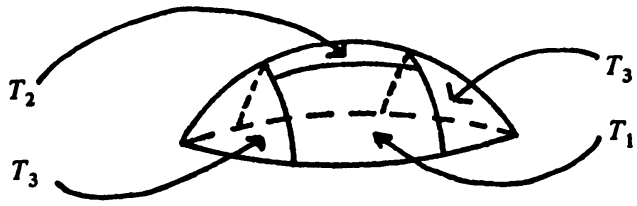

Note that the induced $T^{3}$-actions on the boundaries, $S^{2} \times S^{3}$, are equivalent.

Now suppose we have a $T^{3}$-manifold $M$ with orbit space $M^{*} \cong D^{3}$ as follows:

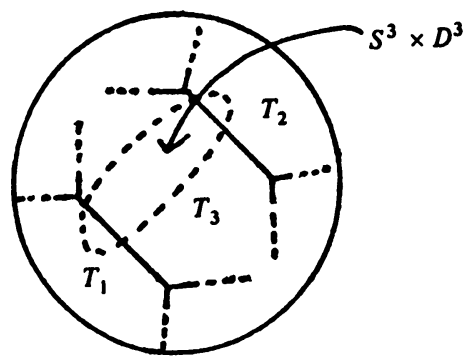

From results in $\S 6$ we know the indicated region must be $S^{3} \times D^{3}$. Hence we may do equivariant surgery on it, replacing it with $S^{2} \times D^{4}$, to obtain a new manifold $M^{\prime}$ with orbit space:

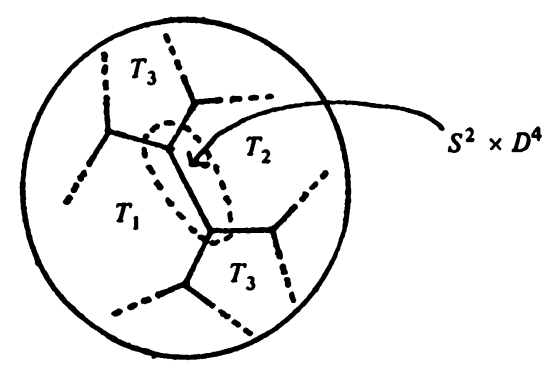

Note that we have introduced two more fixed points.

4. Orbit structure. We now investigate the orbit structure of a $T^{3}$-action. We first consider conical orbit structures.

Let $\overline{C B}=(B \times I) /(B \times 0)$ be the closed cone over a space $B$ and $C B=$ $(B \times[0,1)) /(B \times 0)$ the open cone. Let $X$ be a $G$-space with orbit space $Y$.

4.1 Definition. The orbit structure for $X$ is conical if $Y$ is homeomorphic to an open cone $C B$ with orbit type constant along rays (less the vertex).

4.2 Proposition (Corollary 8.5, Chapter II of [1]). Let $M$ be a G-manifold, $G$ compact Lie. Suppose that the orbit structure is conical with vertex $x_{0}^{*}$, 
and that there is a slice $V$ at $x_{0}$ which has the form of Euclidean space with a linear $G_{x_{0}}$-action. Then $M$ is equivalent as a $G$-space to $G \times{ }_{x_{x_{0}}} V$.

We shall often be concerned with torus actions where the orbit space is a closed cone. In this case we have the following:

4.3 THEOREM. Suppose $T^{n}$ acts locally smoothly on a closed compact manifold $M$ of dimension $m>n$. Suppose $x_{0} \in M$ has stability group $T^{k}, 0 \leqslant$ $k \leqslant n\left(T^{0}=e\right.$, the identity element). Let $X$ be a closed invariant neighborhood of $x_{0}$ such that $X^{*}=\overline{C B}$ with vertex $x_{0}^{*}$. Suppose $C B$ is an open subspace of $M^{*}$. Then $X$ is equivariantly homeomorphic to $T^{n-k} \times D^{m-n+k}$.

Proof. See [11].

Now suppose $M$ is a compact, connected 6-manifold and $T^{3}$ acts on $M$. We derive some elementary facts about the orbit structure of such an action.

By 3.1, Chapter IV of [1], there exists a maximal orbit type $T^{3} / H$. Furthermore, if $M_{(H)}$ is the union of orbits of this type, then $M_{(H)}$ is open and dense in $M$ and its image, $\left(M_{(H)}\right)^{*}$, in $M^{*}=M / T^{3}$ is connected. The maximal orbits are called principal. The following lemma is elementary.

4.4 Lemma. Principal orbits are of type $T^{3}$.

Now suppose $M$ is closed and simply connected, and the only stability groups are torus subgroups of $\mathrm{T}^{3}$. By 4.7 , Chapter IV of [1], $M^{*}$ is a simply connected 3-manifold, with or without boundary.

4.5 LEMma. Points on the boundary of $M^{*}$ are orbits of isotropy type $T^{1}$, $T^{2}$, or $T^{3}$. Interior points are principal orbits.

Proof. This follows from the existence of tubes about an orbit.

In addition to the above assumptions, suppose $M^{*}$ is a 3-manifold with boundary. By examining the linear tubes mentioned above, it is easy to see that the weighted orbit space $M^{*}$ can be described by a graph $G$ on the boundary of $M^{*}$. The vertices will be fixed points, the edges will be orbits with $T^{2}$-stability groups, and the remaining points on $\partial M^{*}$ will be orbits with $T^{1}$-stability groups. The interior points will be principal orbits. We shall call $G$ the orbit structure graph.

We will be primarily concerned with the following type of situation.

4.6 Definition. A $T^{3}$-action will be called regular if every stability group is a torus subgroup of $T^{3}$. We say it is $F$-regular if every component of $G$ contains a fixed point.

Now suppose $T^{\mathbf{3}}$ acts in a locally smooth manner on a compact 6-manifold $M$ with $\omega_{2}(M)=0$. Suppose $N$ is a closed subspace of $M$ such that $N^{*}$ is as shown: 


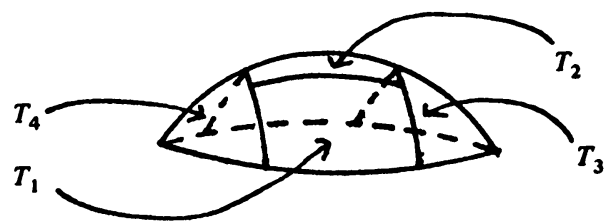

$N$ can be obtained by attaching two copies of $D^{6}$ together via an equivariant homeomorphism $f: T^{1} \times D^{4} \rightarrow T^{1} \times D^{4}$ as shown:

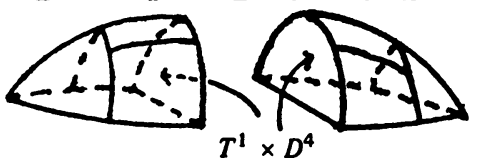

Hence, we obtain a $D^{4}$-bundle over $S^{2}$. Since $\omega_{2}(M)=0$, this must be trivial and hence $S^{2} \times D^{4}$.

We wish to show that the condition $\omega_{2}(M)=0$ imposes strong conditions on the stability group $T_{4}$. Throughout this discussion we parameterize $T^{3}$ as $T^{3}$ $=\left\{\left(e^{i \varphi_{1}}, e^{i \varphi_{2}}, e^{i \varphi_{3}}\right) \mid 0 \leqslant \varphi_{i} \leqslant 2 \pi\right\}=T_{1} \times T_{2} \times T_{3}$. We have the standard orthogonal action of $T^{3}$ on $D^{6},\left(T^{3}, D_{1}^{6}\right)$, defined in 3.1 with circle stability groups $T_{1}, T_{2}$ and $T_{3}$. Now suppose we have another orthogonal action $\left(T^{3}, D_{2}^{6}\right)$ with circle stability groups $T_{1}, T_{2}$ and $T_{4}$. This action can be interpreted as a three-dimensional complex representation of $T^{3}$ and hence (see [13]), the action can be defined by:

$$
\begin{array}{r}
t z=\left(r_{1} e^{i\left(\theta_{1}+a_{1} \varphi_{1}+a_{2} \varphi_{2}+a_{3} \varphi_{3}\right)}, r_{2} e^{i\left(\theta_{2}+b_{1} \varphi_{1}+b_{2} \varphi_{2}+b_{3} \varphi_{3}\right)},\right. \\
\left.r_{3} e^{i\left(\theta_{3}+c_{1} \varphi_{1}+c_{2} \varphi_{2}+c_{3} \varphi_{3}\right)}\right)
\end{array}
$$

where $z=\left(r_{1} e^{i \theta_{1}}, r_{2} e^{i \theta_{2}}, r_{3} e^{i \theta_{3}}\right) \in D_{2}^{6}$. For $T_{1}$ and $T_{2}$ to be stability groups we may assume that $b_{1}=c_{1}=0$. For the action to be effective it is easy to see that $a_{1}=b_{2}=c_{3}= \pm 1$ (we assume +1 for convenience). Therefore our action $\left(T^{3}, D_{2}^{6}\right)$ is defined by

$$
t z=\left(r_{1} e^{i\left(\theta_{1}+\varphi_{1}+a_{3} \varphi_{3}\right)}, r_{2} e^{i\left(\theta_{2}+\varphi_{2}+b_{3} \varphi_{3}\right)}, r_{3} e^{i\left(\theta_{3}+\varphi_{3}\right)}\right) .
$$

Our third circle stability group is $T_{4}=\left\{t \in T^{3} \mid \varphi_{1}+a_{3} \varphi_{3} \equiv 0(2 \pi) ; \varphi_{2}+\right.$ $\left.b_{3} \varphi_{3} \equiv 0(2 \pi)\right\}$.

Let $\left(T^{1} \times D^{4}\right)_{i}=\left\{z \in \partial D_{i}^{6} \mid r_{3} \geqslant 1 / 2\right\} \subseteq D_{i}^{6}$. Define $f:\left(T^{1} \times D^{4}\right)_{1} \rightarrow$ $\left(T^{1} \times D^{4}\right)_{2}$ by $f(z)=\left(r_{1} e^{i\left(\theta_{1}+a_{3} \theta_{3}\right)}, r_{2} e^{i\left(\theta_{2}+b_{3} \theta_{3}\right)}, r_{3} e^{i \theta} 3\right)$. It is easy to see that $f$ is a homeomorphism and that $f$ is equivariant. We then obtain the action $\left(T^{3}, D_{1}^{6} \cup_{f} D_{2}^{6}\right)$ with orbit space 4.7 , where $T_{4}$ is as above.

\subsection{Lemma. $D_{1}^{6} \cup_{f} D_{2}^{6}=S^{2} \times D^{4}$ iff $a_{3}+b_{3}$ is even.}

Proof. We know $D_{1}^{6} \cup_{f} D_{2}^{6}$ is a $D^{4}$-bundle over $S^{2}$. It will be trivial iff the map $\bar{f}: T^{1} \rightarrow R_{4}$ defined by $\bar{f}(t)=f(t, \ldots)$ represents the trivial element of $\pi_{1}\left(R_{4}\right)$, where $R_{4}$ is the rotation group of $R^{4}$.

A generator of $\pi_{1}\left(R_{4}\right)$ is given by the one parameter family of matrices 


$$
\left(\begin{array}{rrr}
\cos \theta & -\sin \theta & 0 \\
\sin \theta & \cos \theta & \\
& 0 & I
\end{array}\right), \quad 0 \leqslant \theta \leqslant 2 \pi,
$$

and this has order two. The loop $\bar{f}$ in $R_{4}$ is given by the family of matrices below, where $0 \leqslant \theta_{3} \leqslant 2 \pi$.

$$
\left(\begin{array}{cccc}
\cos \left(a_{3} \theta_{3}\right) & -\sin \left(a_{3} \theta_{3}\right) & \\
\sin \left(a_{3} \theta_{3}\right) & \cos \left(a_{3} \theta_{3}\right) & 0 \\
0 & & \cos \left(b_{3} \theta_{3}\right) & -\sin \left(b_{3} \theta_{3}\right) \\
0 & & \sin \left(b_{3} \theta_{3}\right) & \cos \left(b_{3} \theta_{3}\right)
\end{array}\right) \text {. }
$$

This is homotopic to the family

$$
\left(\begin{array}{ccc}
\cos \left(a_{3}+b_{3}\right) \theta_{3} & -\sin \left(a_{3}+b_{3}\right) \theta_{3} & \\
\sin \left(a_{3}+b_{3}\right) \theta_{3} & \cos \left(a_{3}+b_{3}\right) \theta_{3} & 0 \\
0 & I
\end{array}\right), \quad 0 \leqslant \theta_{3} \leqslant 2 \pi .
$$

Hence $\bar{f}$ represents the trivial element of $\pi_{1}\left(R_{4}\right)$ iff $a_{3}+b_{3}$ is even.

4.9 Lemma. Suppose we have an action $\left(T^{3}, S^{2} \times D^{4}\right)$ with orbit space

\subsection{Then}

$$
\begin{array}{r}
T_{4}=\left\{t \in T^{3} \mid \varphi_{1}+a_{3} \varphi_{3} \equiv 0(2 \pi), \varphi_{2}+b_{3} \varphi_{3} \equiv 0(2 \pi) ; a_{3}+b_{3}\right. \text { even; } \\
\left.a_{3}, b_{3}=0 \text { or } \pm 1\right\} .
\end{array}
$$

Proof. We must show that $a_{3}$ and $b_{3}$ can only assume the values 0 or \pm 1 . Consider the induced action $\left(T^{3}, \partial\left(S^{2} \times D^{4}\right)\right)=\left(T^{3}, S^{2} \times S^{3}\right)$. Let $U=F\left(T_{1}, S^{2} \times S^{3}\right), \quad U_{1}=F\left(T_{1} \times T_{4}, S^{2} \times S^{3}\right), \quad U_{2}=F\left(T_{1} \times T_{3}, S^{2} \times S^{3}\right)$.

Then $U_{i} \cong S^{1}, i=1,2$. By restriction we have an effective action $\left(T_{2} \times T_{3}, U\right)$. We have a stability group $T_{3}$ with $F\left(T_{3}, U\right)=U_{2}$ and a stability group $T_{4}^{\prime}=$ $\left\{\left(e^{i \varphi_{2}}, e^{i \varphi_{3}}\right) \mid a_{3} \varphi_{3} \equiv 0(2 \pi) ; \varphi_{2}+b_{3} \varphi_{3} \equiv 0(2 \pi)\right\}$. Then $F\left(T_{4}^{\prime}, U\right)=U_{1} \cong S^{1}$. But if $a_{3} \neq 0$ or $\pm 1, T_{4}^{\prime}$ is finite, and the dimension of $F\left(T_{4}^{\prime}, U\right)$ would be 2 . In a similar manner we see that $b_{3}=0$ or \pm 1 .

4.10 Proposition. Suppose $T^{3}$ acts on a compact 6-manifold $M$, with $\omega_{2}(M)=0$. Suppose $N \subseteq M$ is a closed invariant subspace with $N^{*}$ as in 4.7 . Then $N \cong \cong_{\mathrm{eq}} S^{2} \times D^{4}$ and $T_{4}$ is as in 4.9 .

4.11 CoRollary. Suppose $T^{3}$ acts on a compact 6-manifold with boundary $M$, with $\omega_{2}(M)=0$, such that the orbit space $M^{*}$ is as shown: 


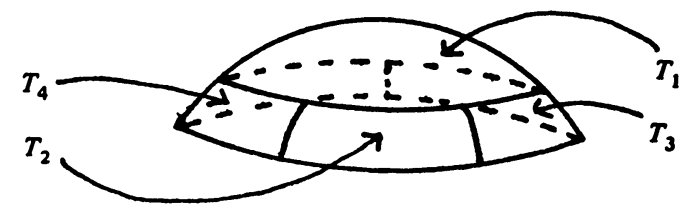

Then $M \cong \cong_{\mathrm{eq}} C P(3)-D^{6}$.

Proof. By 4.10, $T_{4}$ must be as in 4.9. Let

$$
\left.C P(3)=\left.\left\{\bar{z}=\overline{\left(r_{1} e^{i \theta} 1, r_{2} e^{i \theta} 2, r_{3} e^{i \theta} 3, r_{4} e^{i \theta} 4\right.}\right)\right|_{z} \in S^{7}\right\} .
$$

For $t \in T^{3}$ define

$$
t \bar{z}=\overline{\left(r_{1} e^{i\left(\theta_{1}+\gamma_{1} \varphi_{1}+\alpha_{3} a_{3} \varphi_{3}\right)}, r_{2} e^{i\left(\theta_{2}+\gamma_{2} \varphi_{2}+\beta_{3} b_{3} \varphi_{3}\right)},\right.}, \frac{\left.r_{3} e^{i\left(\theta_{3}+\varphi_{3}\right)}, r_{4} e^{i \theta_{4}}\right)}{.}
$$

Note that if either $a_{3}$ or $b_{3}$ is zero, the other is also since $a_{3}+b_{3}$ is even. If $a_{3}, b_{3} \neq 0$, choose $\alpha_{3}$ and $\beta_{3}$ so that $\alpha_{3} a_{3} \varphi_{3}=\varphi_{3}$, and $\beta_{3} b_{3} \varphi_{3}=\varphi_{3}$. Then choose $\gamma_{1}$ and $\gamma_{2}$ so that

$$
\gamma_{1} \varphi_{1}+\alpha_{3} a_{3} \varphi_{3}= \pm\left(\varphi_{1}+a_{3} \varphi_{3}\right), \quad \gamma_{2} \varphi_{2}+\beta_{3} b_{3} \varphi_{3}= \pm\left(\varphi_{2}+b_{3} \varphi_{3}\right)
$$

Then this action :.as stability groups $T_{1}, T_{2}, T_{3}$ and $T_{4}$. The orbit space $(C P(3)) *$ is as shown below.

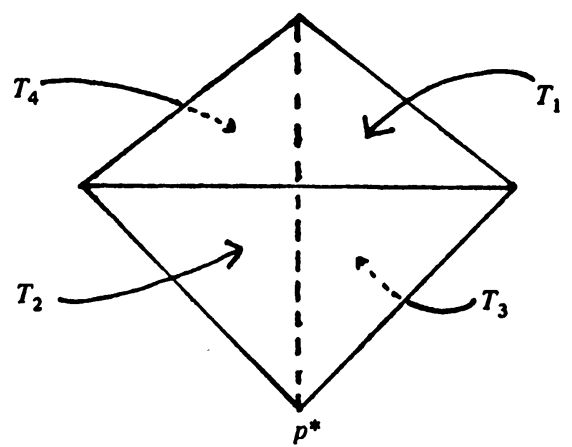

By removing an invariant neighborhood of $p$ homeomorphic to $D^{6}$, we obtain a $T^{3}$-action on $C P(3)-D^{6}$ with orbit space identical to $M^{*}$. Hence, by results from $\S 6, M \cong \cong_{\mathrm{eq}} C P(3)-\stackrel{D}{ }^{6}$.

4.12 EXAMPLE. We now construct another $T^{3}$-action on $S^{4} \times S^{2}$. Let $a=$ 0 or \pm 1 and $b=0$ or \pm 1 , with $a+b$ even. Define two actions $\left(T^{3},\left(S^{4} \times D^{2}\right)_{1}\right)$ and $\left(T^{3},\left(S^{4} \times D^{2}\right)_{2}\right)$ as follows:

$$
\begin{gathered}
t z_{1}=\left(\left(r_{0}, r_{1} e^{i\left(\theta_{1}+\varphi_{1}\right)}, r_{2} e^{i\left(\theta_{2}+\varphi_{2}\right)}\right),\left(r_{3} e^{i\left(\theta_{3}+\varphi_{3}\right)}\right)\right), \\
t z_{2}=\left(\left(r_{0}, r_{1} e^{i\left(\theta_{1}+\varphi_{1}+\varphi_{3}\right)}, r_{2} e^{i\left(\theta_{2}+\varphi_{2}+b \varphi_{3}\right)}\right),\left(r_{3} e^{i\left(\theta_{3}+\varphi_{3}\right)}\right)\right),
\end{gathered}
$$


where $z_{i} \in\left(S^{4} \times D^{2}\right)_{i}$. The first action has circle stability groups $T_{1}, T_{2}$ and $T_{3}$; the second has circle stability groups $T_{1}, T_{2}$ and $T_{4}$. We attach $\left(S^{2} \times D^{4}\right)_{1}$ to $\left(S^{2} \times D^{4}\right)_{2}$ via an equivariant homeomorphism $f:\left(S^{4} \times S^{1}\right)_{1} \rightarrow\left(S^{4} \times S^{1}\right)_{2}$ defined by

$$
f\left(z_{1}\right)=\left(\left(r_{0}, r_{1} e^{i\left(\theta_{1}+a \theta_{3}\right)}, r_{2} e^{i\left(\theta_{2}+b \theta_{3}\right)}\right),\left(r_{3} e^{i \theta_{3}}\right)\right) .
$$

In this way we obtain an action on an $S^{4}$-bundle over $S^{2}$ with orbit space:

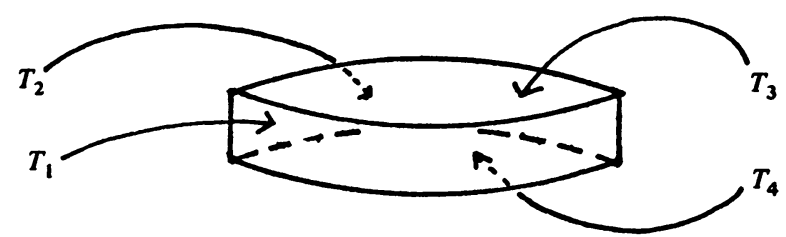

As in $4.8, f$ induces an $\bar{f}$ representing the trivial element of $\pi_{1}\left(R_{5}\right)$. Hence we have the trivial bundle $S^{4} \times S^{2}$.

4.13 ExAMPLE. Let $a$ and $b$ be as in 4.12 except that $a, b \neq 0$. Then we can write $T_{4}$ as $T_{4}=\left\{t \in T^{3} \mid \varphi_{3}+(1 / a) \varphi_{1} \equiv 0(2 \pi) ; \varphi_{2}-(b / a) \varphi_{1} \equiv 0(2 \pi)\right\}$. For $D^{3}=\left\{\left(r_{0}, r_{1} e^{i \theta_{1}}\right) \mid r_{0}^{2}+r_{1}^{2} \leqslant 1\right\}$ and $S^{3}=\left\{\left(r_{2} e^{i \theta_{2}}, r_{3} e^{i \theta}\right) \mid r_{2}^{2}+r_{3}^{2}=1\right\}$ we obtain an action $\left(T^{3}, D^{3} \times S^{3}\right)$ defined by

$$
t z=\left(\left(r_{0}, r_{1} e^{i\left(\theta_{1}+\varphi_{2}-(b / a) \varphi_{1}\right)}\right),\left(r_{2} e^{i\left(\theta_{2}+\varphi_{1}\right)}, r_{3} e^{i\left(\theta_{3}+\varphi_{3}-(1 / a) \varphi_{1}\right)}\right)\right)
$$

where the orbit space is shown below.

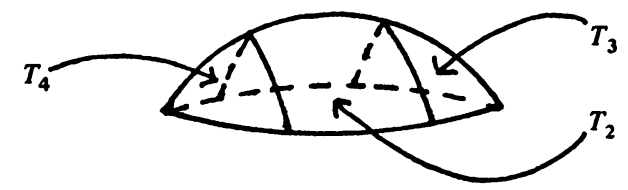

We can easily obtain another action $\left(T^{3}, D^{3} \times S^{3}\right)$ where the stability group $T_{2}$ is replaced by the stability group $T_{1}$.

5. A $T^{3}$-action on $S^{2} \times S^{4} \# S^{3} \times S^{3} \# S^{2} \times S^{4}$. Consider the $T^{3}$-action on $S^{6}$ given in 3.1 with circle stability groups $T_{1}, T_{2}$ and $T_{3}$. Remove the interior of a subspace $V_{1}$ as shown to obtain $U_{1}=S^{6}-\stackrel{\circ}{V}_{1}$.

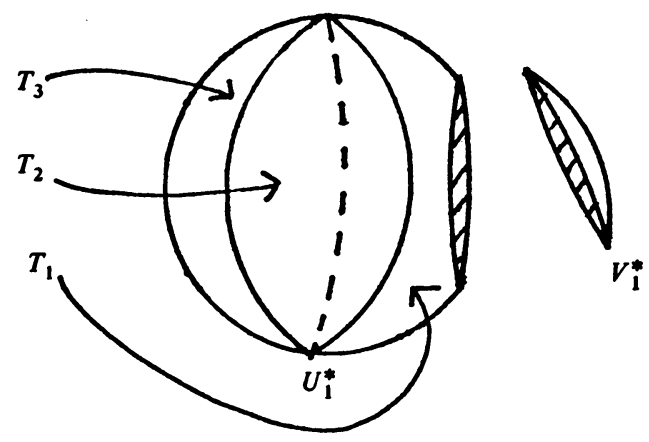


The orbit structure for $V_{1}$ is conical so, by $4.3, V_{1} \cong T^{2} \times D^{4}$. By the MayerVietoris sequence, it is easy to see that

$$
H_{q}\left(U_{1}\right), H^{q}\left(U_{1}\right) \cong \begin{cases}0, & q=1,2,5,6, \\ \mathrm{Z}, & q=0,3, \\ \mathrm{Z} \oplus \mathrm{Z}, & q=4 .\end{cases}
$$

Now suppose we have another $T^{3}$-action on $S^{6}$ as in 3.1, where one of the circle stability groups is $T_{1}$. We may form $U_{2}$ and $V_{2}$ as before where $V_{2} \cap F\left(T_{1}, S^{6}\right)$ $\neq \varnothing$. Then let $M=U_{1} U_{f} U_{2}$, where $f$ is an equivariant homeomorphism from $\partial U_{1}$ to $\partial U_{2}$. In this way, we obtain an action $\left(T^{3}, M\right)$ with orbit space:

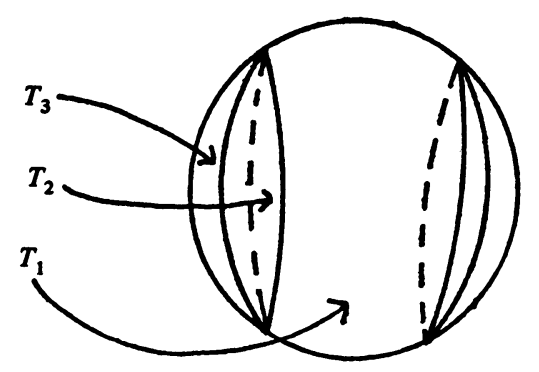

We wish to show that $M=S^{2} \times S^{4} \# S^{3} \times S^{3} \# S^{2} \times S^{4}$.

By Van Kampen's theorem, $M$ is simply connected. From the Mayer-Vietoris sequence, we can see that

$$
H_{q}(M), H^{q}(M) \cong \begin{cases}0, & q=1,5, \\ \mathrm{Z}, & q=0,6, \\ \mathrm{Z} \oplus \mathrm{Z}, & q=2,3,4 .\end{cases}
$$

We must compute the ring structure for $H^{*}(M)$. By [20], we know that $M=M_{1} \# S^{3} \times S^{3}$. Hence, if $\alpha_{3}$ and $\beta_{3}$ are the generators of $H^{3}(M)$, then $\alpha_{3}$ $\cup \beta_{3}=\alpha_{6}$ is a generator of $H^{6}(M)$ and $\alpha_{3}^{2}=\beta_{3}^{2}=0$.

We now compute the other products. First, note that the ring $H^{*}\left(U_{1} \cap U_{2}\right)$ $\cong H^{*}\left(S^{3} \times T^{2}\right)$ is given by generators $\alpha$ and $\beta$ of degree one and $\gamma$ of degree three, where $\alpha \cup \beta$ generates $H^{2}\left(U_{1} \cap U_{2}\right), \alpha \cup \gamma$ and $\beta \cup \gamma$ generate $H^{4}\left(U_{1} \cap U_{2}\right)$, and $\alpha \cup \beta \cup \gamma$ generates $H^{5}\left(U_{1} \cap U_{2}\right)$. We have the isomorphism $\delta_{1}^{*}: H^{1}\left(U_{1} \cap U_{2}\right) \rightarrow H^{2}(M)$, so let $\alpha_{2}=\delta_{1}^{*}(\alpha)$ and $\beta_{2}=\delta_{1}^{*}(\beta)$. The inclusion $k: U_{1} \cap U_{2} \rightarrow M$ induces an isomorphism $k^{*}: H^{4}(M) \rightarrow H^{4}\left(U_{1} \cap U_{2}\right)$. Therefore, choose $\alpha_{4}$ and $\beta_{4}$ so that $k^{*}\left(\alpha_{4}\right)=\beta \cup \gamma$ and $k^{*}\left(\beta_{4}\right)=-\alpha \cup \gamma$. Now, since $\delta_{5}^{*}: H^{5}\left(U_{1} \cap U_{2}\right) \rightarrow H^{6}(M)$ is an isomorphism, we have, by the MayerVietoris sequence:

$$
\begin{gathered}
\alpha_{2} \cup \alpha_{4}=\delta_{1}^{*}(\alpha) \cup \alpha_{4}=\alpha_{6}, \quad \alpha_{2} \cup \beta_{4}=\beta_{2} \cup \alpha_{4}=0, \\
\beta_{2} \cup \beta_{4}=\delta_{1}^{*}(\beta) \cup \beta_{4}=\alpha_{6} .
\end{gathered}
$$


Hence, $H^{*}(M)$ is given by generators $\alpha_{i}$ and $\beta_{i}$ of degree $i, i=2,3,4$, where $\alpha_{2}$ $\cup \alpha_{4}=\beta_{2} \cup \beta_{4}=\alpha_{3} \cup \beta_{3}=\alpha_{6}$ generates $H^{6}(M)$, and all other products involving generators (except permutations of these) are 0 .

By starting with two embeddings of $S^{6}$ in $\mathrm{R}^{8}$, it is not difficult to see that $M$ embeds in $\mathbf{R}^{8}$. By Whitney Duality, $W(M) \cdot \bar{W}(M)=1$, where $W(M)$ is the total Stiefel-Whitney class of $M$, and $\bar{W}(M)$ is the total Stiefel-Whitney class of the normal bundle, $\nu^{2}$, of $M \subseteq \mathbf{R}^{8}$. Since $M$ embeds in $\mathbf{R}^{8}, \bar{\omega}_{i}(M)=0$ for $i \geqslant 2$ and obviously $\bar{\omega}_{1}(M)=0$. Therefore $W(M)=1$ and $\omega_{2}(M)=0$.

Again, since $M$ embeds in $\mathbf{R}^{8}, p_{1}\left(\nu^{2}\right)=\left(\chi\left(v^{2}\right)\right)^{2}=0$. Letting $\tau^{6}$ be the tangent bundle of $M$, we have $\tau^{6} \oplus \nu^{2}=\epsilon^{8}$, the trivial bundle over $M$. Hence,

$$
1=p\left(\epsilon^{8}\right)=p\left(\tau^{6} \oplus \nu^{2}\right)=p\left(\tau^{6}\right) p\left(\nu^{2}\right)+\alpha=p\left(\tau^{6}\right)+\alpha \quad \text { (see [12]) }
$$

where $|\alpha|=2$. Hence, $\alpha=0, p\left(\tau^{6}\right)=1$, and $p_{1}\left(\tau^{6}\right)=p_{1}(M)=0$.

Now it is rather easy to see that the invariants for $M$ are the same as those for $S^{2} \times S^{4} \# S^{3} \times S^{3} \# S^{2} \times S^{4}$. Hence, by $1.1, M \cong S^{2} \times S^{4} \# S^{3} \times S^{3} \#$ $S^{2} \times S^{4}$.

6. Equivariant classification theorems. Throughout this section we assume all actions are locally smooth. We let $\partial D^{3} \cong S^{2}=S^{+} \cup S$, where $S^{+}$and $S$ are the upper and lower hemispheres, respectively, of $S^{2}$ and $S^{+} \cap S \cong S^{1}$.

6.1 THEOREM. Suppose we have a regular $T^{3}$-action on a closed, compact 6-manifold, $M$, with orbit space $M^{*} \cong D^{3}$. Then there is a cross-section.

PROOF. We first decompose the orbit space $M^{*} \cong D^{3}$ into a collection of conical sections $\left\{C_{i}^{*}\right\}$, part of which is shown below:

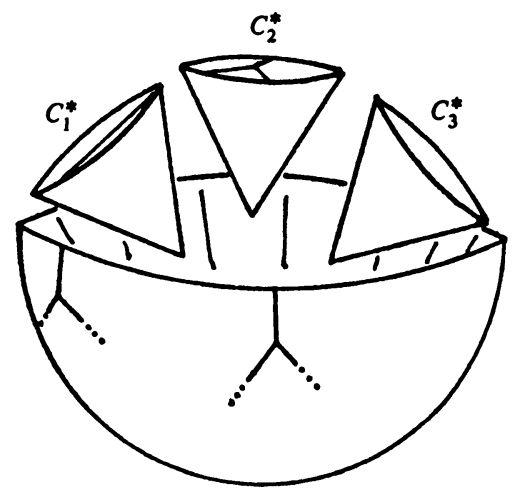

The orbit structure for each $C_{i}$ is conical and hence each $C_{i} \cong T^{3-k} \times D^{3+k}$ for some $k=1,2,3$ (where $T^{0}=$ identity element). It is easy to see that a crosssection would exist on each $C_{i}$. Furthermore, an obstruction theory argument shows that a cross-section given on a 2 -cell $A \subseteq S \subseteq C_{i}$ can be extended to all of $C_{i}$. 
We begin by defining a cross-section $s$ on $C_{1}^{*}$. We then attach $C_{2}^{*}$ to $C_{1}^{*}$ along a 2-cell $A \subseteq S^{-} \subseteq C_{2}^{*}$. We then know how to define $s$ on $A$ and $s$ can be extended to all of $C_{2}^{*}$. Continuing this process we can extend $s$ to all of $M^{*}$.

6.2 THEOREM. Suppose we have two regular actions $\left(T^{3}, M_{1}\right)$ and $\left(T^{3}, M_{2}\right)$ where each $M_{i}$ is a closed, compact 6-manifold. Also suppose there is a weight preserving homeomorphism $h^{*}: M_{1}^{*} \rightarrow M_{2}^{*}$. Then there is an equivariant homeomorphism $h: M_{1} \rightarrow M_{2}$.

PROof. This follows from 3.3, Chapter 1, of [1].

We comment for future reference that the same results hold if $M$ is a compact, connected 6-manifold with boundary and $M^{*} \cong D^{3}$ with all points in $D^{3}$ $S^{+}$corresponding to principal orbits.

7. Classification theorems. Throughout this section we assume that $M$ is a closed, compact, connected 6-manifold with the second Stiefel-Whitney class $\omega_{2}(M)=0$. We assume that we have a locally smooth, $F$-regular action of $T^{3}$ on $M$ with $M^{*} \cong D^{3}$.

7.1 Proposition. $M$ is an equivariant connected sum of a manifold $\bar{M}$ with copies of $S^{2} \times S^{4}, C P(3)$ and $S^{2} \times S^{4} \# S^{3} \times S^{3} \# S^{2} \times S^{4}$. The orbit space $\bar{M}^{*}$, together with its orbit structure graph $\bar{G}$, has no face with fewer than four sides and $\bar{G}$ is connected.

Proof. Let $G$ be the orbit structure graph for the action $\left(T^{3}, M\right)$. Suppose $G$ has more than one component. Then the orbit space $M^{*}$ may be cut apart as shown.
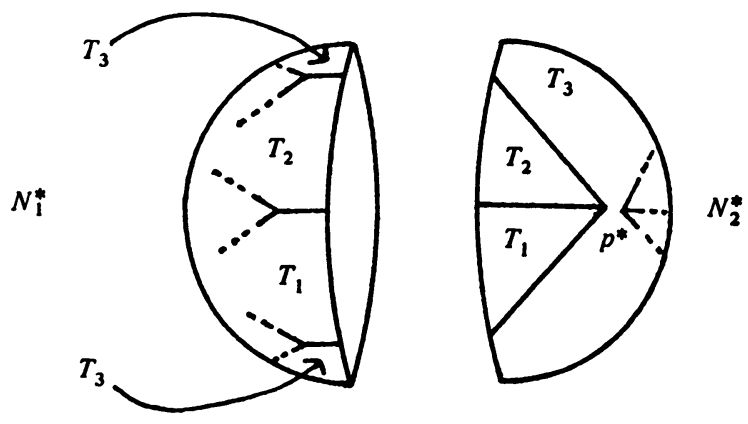

Note that the circle stability groups $T_{1}, T_{2}$ and $T_{3}$ are those occurring in an invariant neighborhood of the fixed point $p$. Hence, we have a $T^{3}$-action on $D^{6}$ with these circle stability groups. The construction in $\$ 3$ shows that $M$ is an equivariant connected sum of $M_{1}$ and $M_{2}$, where each $M_{i}$ is obtained by attaching a $D^{6}$ to $N_{i}$.

Now note that $M_{2}^{*}$ is as shown below: 


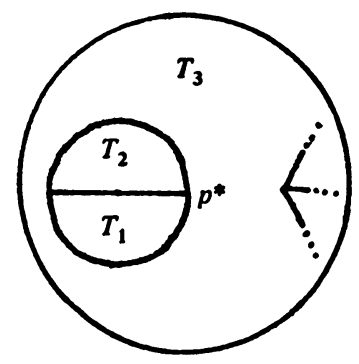

Repeating this process again we see that $M_{2}$ is an equivariant connected sum of $M_{3}$ and $S^{2} \times S^{4} \# S^{3} \times S^{3} \# S^{2} \times S^{4}$, where the action on the latter is the one constructed in $\S 5$.

By continuing this process we eventually obtain $M$ as an equivariant connected sum of a manifold $\bar{M}_{1}$ with copies of $S^{2} \times S^{4} \# S^{3} \times S^{3} \# S^{2} \times S^{4}$, where the orbit structure graph $G_{1}$, for the action $\left(T^{3}, \bar{M}_{1}\right)$ has only one component.

Now suppose $G_{1}$ encloses a region on $\partial\left(\bar{M}_{1}^{*}\right)$ having only two sides. We may then cut $\bar{M}_{1}^{*}$ as shown:
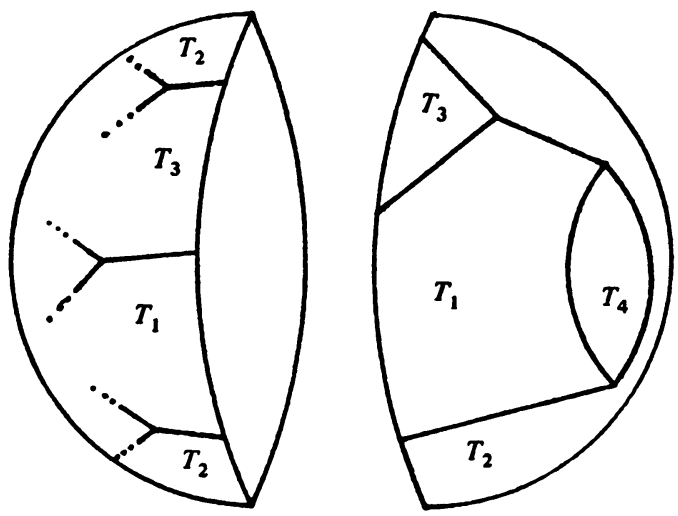

Then, as before, we see that $\bar{M}_{1}$ is an equivariant connected sum of a manifold $\bar{M}_{2}$ and $S^{2} \times S^{4}$, where $\left(T^{3}, S^{2} \times S^{4}\right)$ is the action given in 3.3 or 4.12.

If $G_{1}$ encloses a region on $\partial\left(\bar{M}_{1}^{*}\right)$ having three sides we may cut off a section like that shown in 4.11 and see that $\bar{M}_{1}$ is an equivariant connected sum of a manifold $\bar{M}_{2}$ and $C P(3)$.

By repeating the above constructions we obtain the desired results.

We now assume that the orbit space $M^{*}$, together with the orbit structure graph $G$, has no face with fewer than four sides and that $G$ is connected. Furthermore we assume there are precisely three distinct circle stability groups $T_{1}, T_{2}$ and $T_{3}$ with $T^{3}=T_{1} \times T_{2} \times T_{3}$.

7.2 LEMMA. $M$ may be obtained from $S^{6}$ via equivariant surgery on disjoint copies of $S^{3} \times D^{3}$.

Proof. Starting with $M$, we do the surgery described in $\S 3$ in reverse or- 
der. Since each surgery will reduce the number of fixed points, we will eventually obtain $S^{6}$, as long as the surgery does not disconnect $G$. Note that different $T^{1}$-stability groups must appear on each side of an edge of $G$. Hence $G$ cannot be disconnected by cutting only one edge of $G$. Therefore, if surgery on an $S^{2} \times$ $D^{4}$ disconnects $G$, we must have the following situation:

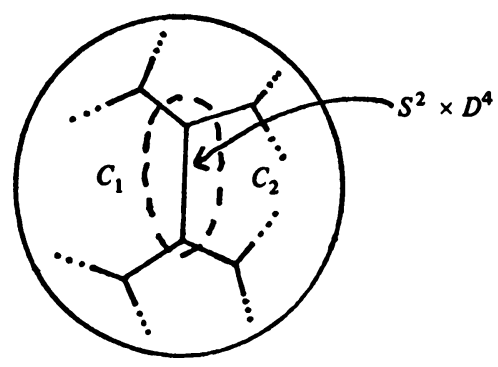

Here, $C_{1}$ and $C_{2}$ indicate the two components of $G$ that would remain if the indicated $S^{2} \times D^{4}$ were removed. It is easy to see that we can choose a different copy of $S^{2} \times D^{4}$ on which to do surgery so that $G$ remains connected. Hence we may obtain $S^{6}$ from $M$ by surgeries on copies of $S^{2} \times D^{4}$. Reversing this process proves the theorem.

We now assume that there are more than three $T^{1}$-stability groups.

7.3 Definition. We shall say that an action $\left(T^{3}, M_{1}\right)$ is obtained from an action $\left(T^{3}, M_{2}\right)$ by a $C S$-operation if $M_{2}$ is obtained from an equivariant connected sum $M_{1} \# K$ by equivariant surgery on an embedded $S^{n} \times D^{6-n}$ intersecting both $M_{1}$ and $K$, where $K$ is an equivariant connected sum of one or two copies of $C P(3)$.

7.4 THEOREM. Suppose the action $\left(T^{3}, M\right)$ has more than three circle stability groups. Then this action can be obtained from an action $\left(T^{3}, M^{\prime}\right)$ by a series of CS-operations, where the action $\left(T^{3}, M^{\prime}\right)$ has only three $T^{1}$-stability groups or $M^{\prime}=\varnothing$.

PROOF. Since there are at least four circle stability groups we have the following situation:

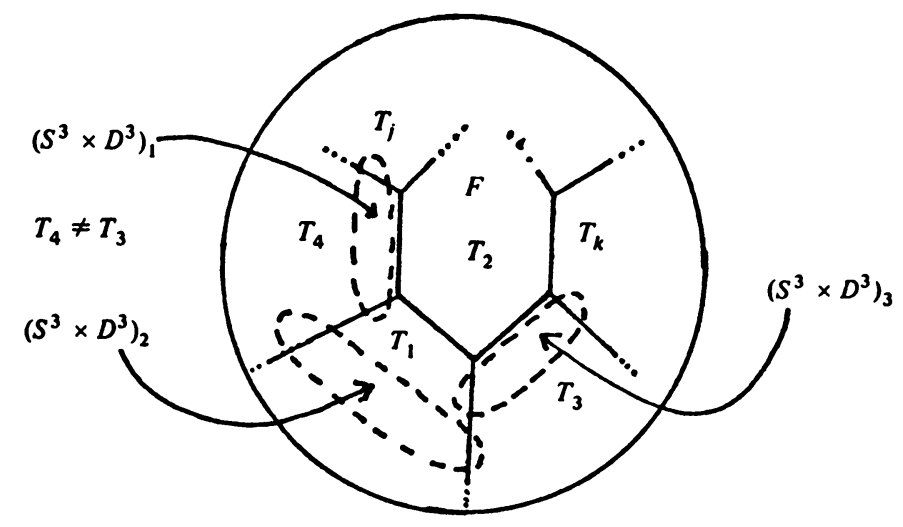


Since $\omega_{2}(M)=0, \S 4$ shows that we have the indicated embeddings of $S^{3} \times D^{3}$. If $T_{k}=T_{4}$ and $T_{j} \neq T_{1}$, we do surgery on $\left(S^{3} \times D^{3}\right)_{1}$; if $T_{k}=T_{4}$ and $T_{j}=T_{1}$, or if $T_{k}=T_{1}$, we do surgery on $\left(S^{3} \times D^{3}\right)_{2}$; if $T_{k} \neq T_{1}, T_{4}$ we do surgery on $\left(S^{3} \times D^{3}\right)_{3}$. We end up with one of the following cases.
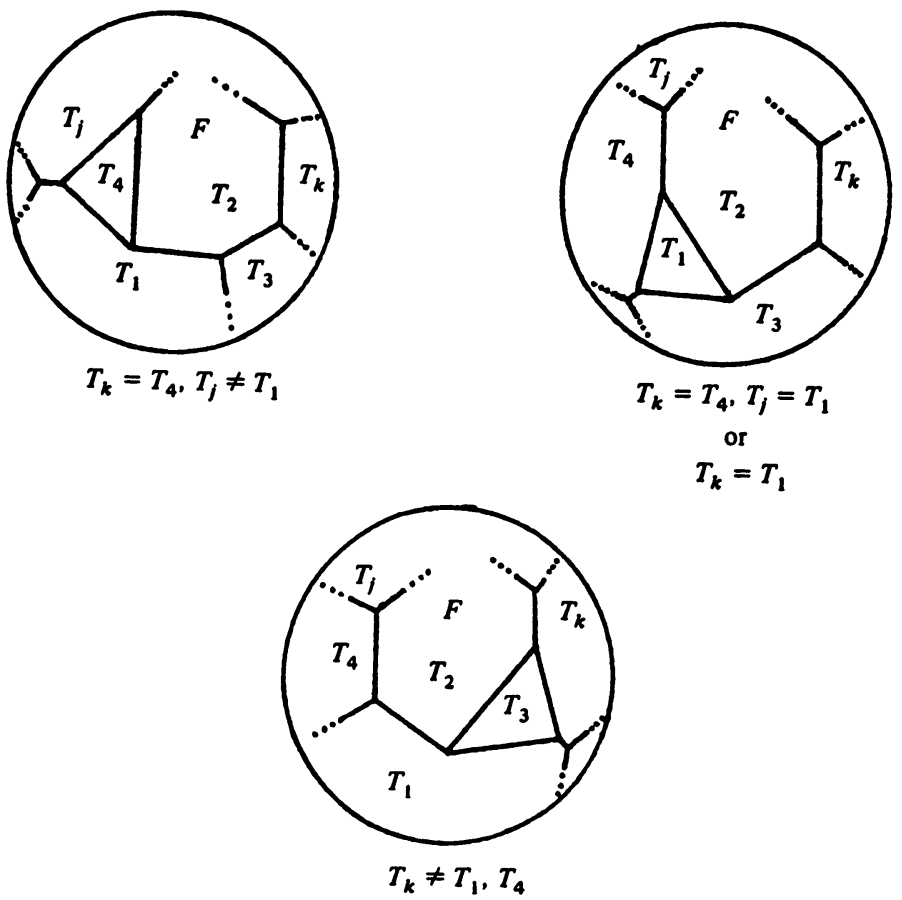

In each case we may remove a $C P(3)$ summand as in $\S 3$. Note that, after doing this, $F$ has one fewer side and we may repeat the process. We do so until $F$ has only three sides, allowing us to remove another $C P(3)$ summand. The resulting orbit space will have one fewer face than $M^{*}$. Continuing in this manner we will eventually obtain the desired $M^{\prime}$.

We continue under the assumptions stated at the beginning of this section. What we have shown so far is that such a $T^{3}$-manifold $M$ is a connected sum of a manifold $M^{\prime}$ and copies of $S^{2} \times S^{4}, S^{2} \times S^{4} \# S^{3} \times S^{3} \# S^{2} \times S^{4}$ and $C P(3)$. Furthermore $M^{\prime}$ can be obtained from $S^{6}$ (or $\varnothing$ ) by a combination of surgery and $C S$-operations. Since the latter do not affect the fundamental group we have the following.

7.5 THEOREM. $M$ is simply connected.

7.6 LEMMA. Suppose $H_{*}(M)$ is torsion-free and $H_{3}(M) \cong 0$. If $N$ is $o b$ tained from $M$ via surgery, then $H_{*}(N)$ is torsion-free.

Proof. This follows by applying the Mayer-Vietoris sequence to the steps in the surgery. 
7.7 Theorem. Suppose $\omega_{2}(M)=0$ and $\left(T^{3}, M\right)$ is a locally smooth, $F$ regular action with $M^{*} \cong D^{3}$. Then $M$ is simply connected and $H_{*}(M)$ is torsionfree.

Proof. 7.6 shows that for the manifold $M^{\prime}$ mentioned in $7.4, H_{*}\left(M^{\prime}\right)$ is torsion-free. It then follows that $H_{*}(M)$ is torsion-free. $M$ is simply connected by 7.5 .

We now compute the ring $H^{*}(M)$ and $p_{1}(M)$ for a $T^{3}$-manifold $M$ satisfying the conditions at the beginning of this section. By 7.1, we may assume that $G$ is connected and that $M^{*}$ has no face with fewer than four sides.

We first assume that the action $\left(T^{3}, M\right)$ has only three distinct $T^{1}$-stability groups. By 7.2, $M$ may be obtained from $S^{6}$ via equivariant surgery. Given the action $\left(T^{3}, S^{6}\right)$ described in 3.1 , the type of surgery we do is shown below:

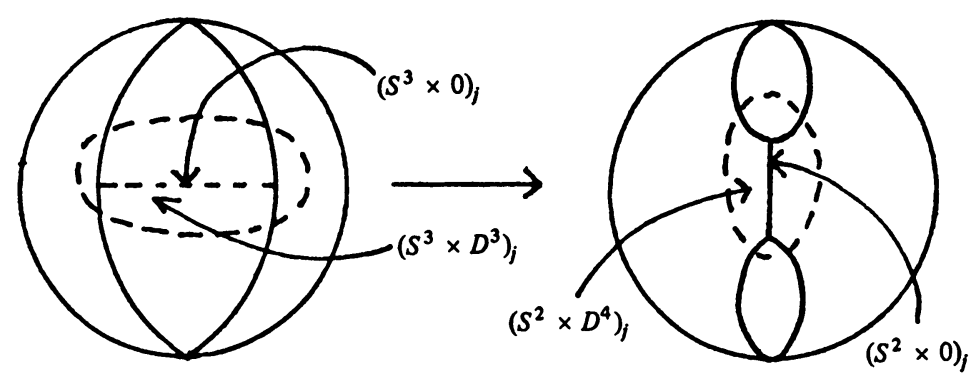

We obtain a basis $\left\{e_{j}\right\} \subseteq H^{2}(M)$, where each $e_{j}$ corresponds to a surgery done on an embedded $\left(S^{3} \times D^{3}\right)_{j}$. The trilinear form $\mu\left(e_{i}, e_{j}, e_{k}\right)=e_{i} \cup e_{j} \cup e_{k}$ and the first Pontrjagin class $p_{1}(M)$ are determined by the embeddings $\left(S^{3} \times 0\right)_{j}=S_{j}^{3}$ as described in $\S 1$. We have shown the surgery being done on a standard embedding of $S^{3} \times D^{3}$ in $S^{6}$. By 7.3, Chapter II of [1], it is easy to see that any equivariantly embedded $S^{3} \times D^{3}$ is isotopic to such an embedding since there is an orbit-type preserving isotopy between their images in the orbit space. Therefore, in the equivariant case, the embeddings $S^{3} \times D^{3} \rightarrow S^{6}$ and $S^{3} \rightarrow S^{6}$ represent the trivial elements in the groups $F C_{3}^{3}$ and $C_{3}^{3}$, respectively. Hence, we have the following.

7.8 Lemma. $\mu\left(e_{i}, e_{i}, e_{i}\right)=0$ for all $i$.

Proof. This follows from 1.3 since $\varphi_{i}=\beta_{i}^{\prime}=0$.

Now suppose we have two basis elements $e_{i}$ and $e_{j}$ for $H^{2}(M)$ corresponding to two embeddings $S_{i}^{3}$ and $S_{j}^{3}$.

7.9 Lemma. $\mu\left(e_{j}, e_{j}, e_{i}\right)=0$ for all $i, j, i \neq j$.

PROOF. By $1.3, \mu\left(e_{j}, e_{j}, e_{i}\right)=\lambda_{j}^{i}$. Hence we must look at the homotopy class of $S_{i}^{3}$ in $S^{6}-S_{j}^{3}$. We may assume $S_{j}^{3}=S_{\alpha}^{3}, S_{i}^{3}=S_{\beta \pm}^{3}$, where $\alpha, \beta=1,2$, 
or 3 (using the notation of $\S 1$ ). Hence, either $r_{4}>0$ for all $z \in S_{i}^{3}$, or $r_{4}<0$ for all $z \in S_{i}^{3}$. In any case, looking at the homotopy equivalence between $S^{6}-$ $S_{\alpha}^{3}$ and $S_{\alpha}^{2}$ constructed in $\S 1$ we see that $S_{\beta \pm}^{3}$ represents the trivial element in $\pi_{3}\left(S_{\alpha}^{2}\right)$ since it is not onto. Therefore $\lambda_{j}^{i}=0$.

As noted in $\S 1, \pi_{3}\left(S^{2} \vee S^{2}\right)=\pi_{3}\left(S^{2}\right) \oplus \pi_{3}\left(S^{2}\right) \oplus \pi_{3}\left(S^{3}\right)$, where the generator of $\pi_{3}\left(S^{3}\right)$ is the Whitehead product of the inclusion maps of the two copies of $S^{2}$. This is given as follows (see [7]). Consider $S^{3}$ as $\left(S^{1} \times D^{2}\right)_{1} U$ $\left(S^{1} \times D^{2}\right)_{2}$, the union of two solid tori attached via an orientation reversing homeomorphism of the boundaries. We wish to define $\sigma: S^{3} \rightarrow S_{+}^{2} \vee S_{-}^{2}$. Let $\sigma\left(t \times D^{2}\right)_{1}$ be a generator of $\pi_{2}\left(S_{+}^{2}\right)$ for all $t$, where $\sigma\left(t \times \partial D^{2}\right)_{1}=(0,-1) \in$ $S_{+}^{2}$. Let $\sigma\left(t \times D^{2}\right)_{2}$ be a generator of $\pi_{2}\left(S_{-}^{2}\right)$ for all $t$, where $\sigma\left(t \times \partial D^{2}\right)_{2}=$ $(0,1) \in S_{-}^{2}$. This gives a well-defined, onto map $\sigma: S^{3} \rightarrow S_{+}^{2} \vee S_{-}^{2}$.

To compute $\lambda_{j k}^{i}$, as defined in $\S 1$, we must look at the homotopy class of $S_{i}^{3}$ in $S^{6}-\left(S_{j}^{3} \cup S_{k}^{3}\right)$. The latter is homotopy equivalent to $S_{+}^{2} \vee S_{-}^{2}$, and we project the class of $S_{i}^{3}$ onto the summand $\pi_{3}\left(S^{3}\right)$ generated by $\sigma$. Again we may assume $S_{j}^{3}=S_{\alpha-}^{3}$ and $S_{k}^{3}=S_{\beta+}^{3}$.

7.10 Lemma. For distinct values for $i, j$ and $k, \mu\left(e_{i}, e_{j}, e_{k}\right)=0$ or \pm 1 .

Proof. Suppose $S_{i}^{3}$ is equivariantly homotopic in $S^{6}-\left(S_{\alpha-}^{3} \cup S_{\beta+}^{3}\right)$ to an $S^{3}$ contained entirely in $D_{+}^{6}$ or entirely in $D_{-}^{6}$. Composing $g_{i}$ with the homotopy equivalences constructed in $\S 1$ we get a map $S_{i}^{3} \rightarrow S_{+}^{2} \vee S_{-}^{2}$ that is not onto. Hence $\lambda_{j k}^{i}=0$.

It is easy to see that the only time the above does not occur is when we have the following type of situation:

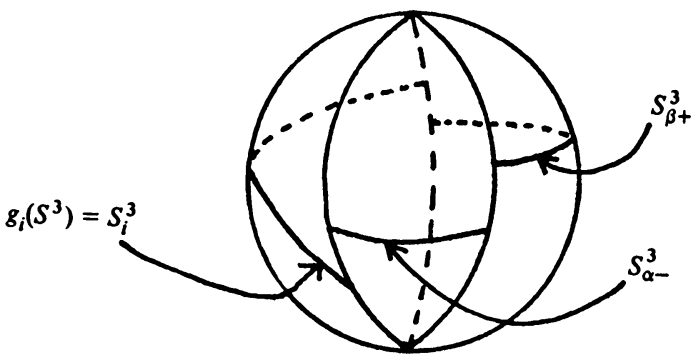

We must show that $g_{i}\left(S^{3}\right)=S_{i}^{3}$ represents $\sigma$ when composed with our homotopy equivalences.

Let us assume that $S_{\beta+}^{3}=S_{3+}^{3}, S_{\alpha-}^{3}=S_{2-}^{3}$. Let $T^{1} \times D^{2}=\left\{\left(e^{i \varphi_{1}}\right.\right.$, $\left.\left.(3 / 4-r) e^{i \varphi_{2}}\right) \mid 0 \leqslant r \leqslant 3 / 4\right\}$. Define homeomorphisms $g_{+}$and $g_{-}$from two copies of $T^{1} \times D^{2},\left(T^{1} \times D^{2}\right)_{+}$and $\left(T^{1} \times D^{2}\right)_{-}$respectively, into $S^{6}$ as follows:

$$
\begin{array}{r}
g_{+}\left(e^{i \varphi_{1}},(3 / 4-r) e^{i \varphi_{2}}\right)=\left(0, \sqrt{1 / 12(6-r)} e^{i \varphi_{1}},\right. \\
\left.\sqrt{1 / 12(3-4 r)(3 r+2)} e^{i \varphi_{2}}, r\right),
\end{array}
$$




$$
\begin{gathered}
g_{-}\left(e^{i \varphi_{1}},(3 / 4-r) e^{i \varphi_{2}}\right)=\left(0, \sqrt{1 / 12(3-4 r)(2+3 r)} e^{i \varphi_{2}},\right. \\
\left.\sqrt{1 / 12(6-r)} e^{i \varphi_{1}},-r\right) .
\end{gathered}
$$

Now $g_{+}=g_{-}$for $r=0$ except that the $\varphi_{i}$ 's are reversed. Hence we can define $g: S^{3}=\left(T^{1} \times D^{2}\right)_{+} \cup\left(T^{1} \times D^{2}\right)_{-} \rightarrow S^{6}$ by

$$
g= \begin{cases}g_{+} & \text {on }\left(T^{1} \times D^{2}\right)_{+}, \\ g_{-} & \text {on }\left(T^{1} \times D^{2}\right)_{-} .\end{cases}
$$

We may assume, without loss of generality, that $g_{i}=g$. It can then be shown that $g_{i}$ composed with our homotopy equivalences gives the desired element in $\pi_{3}\left(S^{2} \vee S^{2}\right)$.

We now have the following.

7.11 THEOREM. Suppose $M$ is a closed, compact, connected 6-manifold with $\omega_{2}(M)=0$. Suppose $\left(T^{3}, M\right)$ is a locally smooth $F$-regular action with $M^{*} \cong D^{3}$. Then $M$ is simply connected. Furthermore $M$ is an equivariant connected sum of a manifold $\bar{M}$ and copies of $S^{2} \times S^{4}, C P(3)$ and $S^{2} \times S^{4} \# S^{3} \times$ $S^{3} \# S^{2} \times S^{4} . \bar{M}$ is a connected sum of copies of $C P(3)$ and a manifold $\overline{\bar{M}}$. The invariants for $\overline{\bar{M}}$ are as follows:

$$
\omega_{2}(\overline{\bar{M}})=p_{1}(\overline{\bar{M}})=0, \quad H^{2}(\overline{\bar{M}}) \cong \mathrm{Z}^{k}=\left\langle e_{1}\right\rangle \oplus \cdots \oplus\left\langle e_{k}\right\rangle .
$$

For distinct integers $i, j$ and $k$ :

$$
\mu\left(e_{i}, e_{i}, e_{i}\right)=\mu\left(e_{i}, e_{i}, e_{j}\right)=0, \quad \mu\left(e_{i}, e_{j}, e_{k}\right)=0 \text { or } \pm 1 .
$$

Proof. By 7.5, $M$ is simply connected. The second statement follows from 7.1. If the action $\left(T^{3}, \bar{M}\right)$ has only three distinct circle stability groups, we are done by the previous three lemmas. It remains to show that if the action $\left(T^{3}, \bar{M}\right)$ has more than three distinct circle stability groups, then $\bar{M}$ can be decomposed as stated. In fact, we need only prove the following:

7.12 Lemma. Suppose $\left(T^{3}, M_{2}\right)$ is obtained by a CS-operation from $\left(T^{3}, M_{1}\right)$. If $M_{1}$ can be decomposed in the desired manner, so can $M_{2}$.

Proof. First note that a $C S$-operation will not affect the second StiefelWhitney class. We first fix our notation. Let $M_{1}^{\prime}$ be $M_{1}$ or $M_{1} \# C P(3)$, depending upon the type of $C S$-operation we do. Let $M_{1}^{\prime \prime}=M_{1}^{\prime} \# C P(3)$. Then $M_{2}$ is obtained from $M_{1}^{\prime \prime}$ by surgery on an embedded $S^{2} \times D^{4}$. Let $N$ be such that $M_{1}^{\prime \prime}=N \cup_{f}\left(S^{2} \times D^{4}\right), M_{2}=N \cup_{g}\left(S^{3} \times D^{3}\right)$ where $f$ and $g$ are the appropriate attaching maps. Now $H^{p}\left(M_{1}^{\prime \prime}\right) \cong H^{p}\left(M_{1}^{\prime}\right) \oplus H^{p}(C P(3))$ for $p=2$, 4. Let $\alpha$ and $\beta=\alpha^{2}$ be generators of $H^{2}(C P(3))$ and $H^{4}(C P(3))$, respectively. We choose a basis $\left\{f_{1}, \ldots, f_{n}\right\}$ for $H^{4}\left(M_{1}^{\prime}\right)$, where $\left\{f_{m}, f_{m+1}, \ldots, f_{n}\right\}$ are the generators corresponding to the $C P(3)$ summands of $M_{1}^{\prime}$. Then $p_{1}\left(M_{1}^{\prime}\right)=f_{m}+\cdots+f_{n}$. 
Also $\left\{f_{1}, \ldots, f_{n}, \beta\right\}$ will be a basis for $H^{4}\left(M_{1}^{\prime \prime}\right)$ and $p_{1}\left(M_{1}^{\prime \prime}\right)=f_{m}+\cdots+f_{n}$ $+\beta$. We show that $M_{2}=M_{1}^{\prime}$ by showing they have the same invariants.

Consider the action $\left(T^{3}, C P(3)\right)$ with orbit space a tetrahedron as shown in the proof of 4.11. Any face corresponds to an embedded $C P(2)$, and hence, corresponds to a generator of $H^{4}(C P(3))$. Then, in $\left(M_{1}^{\prime \prime}\right)^{*}$ (shown below), $F$ represents $\beta$.

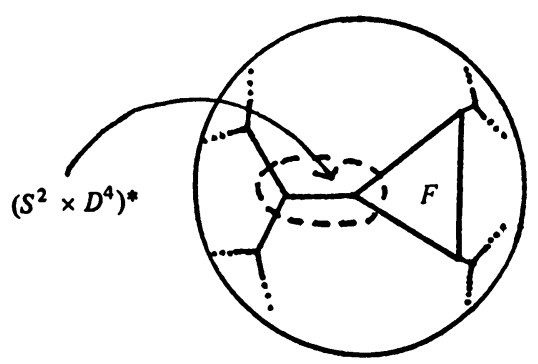

We have the following isomorphisms:

$$
H^{*}\left(M_{1}^{n}, S^{2} \times D^{4}\right) \cong H^{*}\left(N, S^{2} \times S^{3}\right) \cong H^{*}\left(M_{2}, S^{3} \times D^{3}\right)
$$

The appropriate exact sequences can be used to show that:

$$
H^{2}\left(M_{1}^{\prime \prime}, S^{2} \times D^{4}\right) \oplus\langle\alpha\rangle \cong H^{2}\left(M_{1}^{\prime \prime}\right), \quad H^{4}\left(M_{2}\right) \oplus\langle\beta\rangle \cong H^{4}\left(M_{2}, S^{3} \times D^{3}\right)
$$

Roughly speaking, removing the $S^{2} \times D^{4}$ eliminates $\alpha$ and pasting on the $S^{3} \times$ $D^{3}$ eliminates $\beta$. It then follows that we have an isomorphism $H^{*}\left(M_{1}^{\prime}\right) \rightarrow H^{*}\left(M_{2}\right)$ that takes $p_{1}\left(M_{1}^{\prime}\right)$ to $p_{1}\left(M_{2}\right)$. Therefore $M_{1}^{\prime} \cong M_{2}$.

8. An example. We now construct an action $\left(T^{3}, M\right)$ where $p_{1}(M)=0$, $\omega_{2}(M)=0$, and $M$ is not a connected sum of copies of $S^{2} \times S^{4}, S^{3} \times S^{3}$ and manifolds of the form $S^{2} \times\left(S^{2} \times S^{2} \# \cdots \# S^{2} \times S^{2}\right)$.

Starting with the standard action $\left(T^{3}, S^{6}\right)$ we do surgery on embedded $\left(S^{3} \times D^{3}\right)$ 's. It is enough to indicate the zero-sections $\left(S^{3} \times 0\right)$ 's. For our manifold $M$ these are shown below (looking down on the north pole of $\left.\left(S^{6}\right)^{*}\right)$.

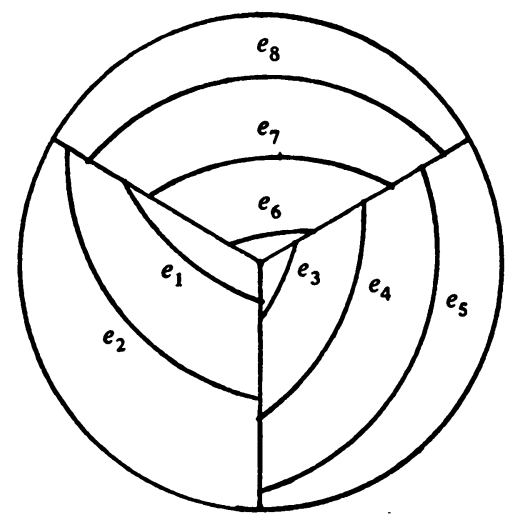


Hence we have a basis $\left\{e_{i}\right\}$ for $H^{2}(M) \cong Z^{8} . p_{1}(M)=\omega_{2}(M)=0$ and, by 7.10, we have:

$$
\begin{aligned}
& \mu\left(e_{1}, e_{3}, e_{6}\right)=\mu\left(e_{1}, e_{3}, e_{7}\right)=\mu\left(e_{1}, e_{4}, e_{7}\right)=1, \\
& \mu\left(e_{2}, e_{4}, e_{7}\right)=\mu\left(e_{2}, e_{4}, e_{8}\right)=\mu\left(e_{2}, e_{5}, e_{8}\right)=1,
\end{aligned}
$$

$\mu \equiv 0$ for any other combination of the $e_{i}$ 's. For convenience, consider the basis $u=\left\{u_{i}\right\}$ where

$$
\begin{array}{llll}
u_{1}=e_{1}, & u_{3}=e_{6}, & u_{5}=e_{7}-e_{6}, & u_{7}=e_{5}, \\
u_{2}=e_{3}, & u_{4}=e_{4}-e_{5}, & u_{6}=e_{2}, & u_{8}=e_{8},
\end{array}
$$

We then have

$$
\mu\left(u_{1}, u_{2}, u_{3}\right)=\mu\left(u_{1}, u_{4}, u_{5}\right)=1, \quad \mu\left(u_{6}, u_{4}, u_{5}\right)=\mu\left(u_{6}, u_{7}, u_{8}\right)=1,
$$

$\mu \equiv 0$ for any other combination of the $u_{i}$ 's. Let $S_{i}=\left[\mu\left(, u_{i},{ }_{-}\right)\right]_{u}$.

Now suppose $M \cong \bar{M} \# S^{2} \times S^{4}$. Let $\alpha \in H^{2}(M)$ be a generator for $H^{2}\left(S^{2} \times S^{4}\right)$. Then $\mu(, \alpha, \ldots) \equiv 0$. However, it is easy to see that for any linear combination $\Sigma_{i=1}^{8} c_{i} u_{i}$,

$$
\mu\left(-, \sum c_{i} u_{i},-\right)=\sum c_{i} \mu\left(-, u_{i},-\right) \neq 0 .
$$

Therefore, if $M$ is a connected sum of the form mentioned above, $M$ must be homeomorphic to

$$
X=S^{2} \times\left(S^{2} \times S^{2} \# S^{2} \times S^{2}\right) \# S^{2} \times S^{2} \times S^{2} .
$$

Hence, let $\eta$ be the trilinear form defined on $H^{2}(X) \cong Z^{8}$. Let $V=\left\{v_{i}\right\}$ be the standard basis for $H^{2}(X)$ with

$$
\eta\left(v_{1}, v_{2}, v_{3}\right)=\eta\left(v_{1}, v_{4}, v_{5}\right)=\eta\left(v_{6}, v_{7}, v_{8}\right)=1,
$$

$\eta \equiv 0$ for any other combination of the $v_{i}$ 's. Let $\bar{S}_{i}=\left[\eta\left(v_{i}, \ldots,-\right)\right]_{v}$.

Suppose $\mu$ and $\eta$ represent the same trilinear form. Then there is a matrix $A=\left(a_{i j}\right)$ and $A^{-1}=B=\left(b_{i j}\right)$ such that

$$
A M^{\prime} A^{T}={ }_{B}^{M}
$$

where $M^{\prime}$ is the matrix for $\eta$ with respect to $V$, and $M$ is the matrix for $\mu$ with respect to $U$. Hence, the slices perpendicular to the $y$-axis in $M$ are the $S_{i}$ 's, and the slices perpendicular to the $x$-axis in $M^{\prime}$ are the $\overline{S_{i}}$ 's. By comparing the columns of these matrices we obtain equations which eventually lead to the following: 


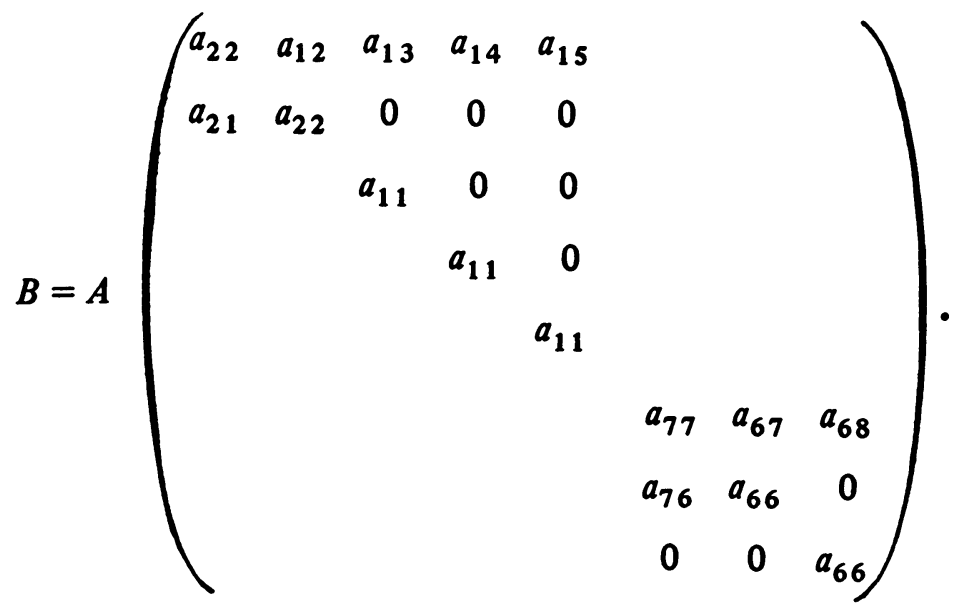

Since $A$ and $B$ represent isomorphisms of $Z$-modules, the determinant of this last matrix must be \pm 1 . Therefore $a_{66}= \pm 1$. Hence

$$
v_{6}= \pm u_{6}+\sum_{i \neq 6} a_{i 6} u_{i}
$$

Then

$$
\eta\left(v_{6},-,-\right)=\mu\left( \pm u_{6}+\sum_{i \neq 6} a_{i 6} u_{i},-,-\right)
$$

Now the rank of $\left[\eta\left(v_{6},-,-\right)\right]_{v}=2$. Therefore the rank of the matrix for $\mu\left( \pm u_{6}+\Sigma_{i \neq 6} a_{i 6} u_{i}, \ldots,-\right)$ must be two in any basis. Choose the basis

$$
W=\left\{ \pm u_{6}+\sum_{i \neq 6} a_{i 6} u_{i}, u_{1}, u_{2}, u_{3}, u_{4}, u_{5}, u_{7}, u_{8}\right\} \text {. }
$$

It is easy to see that the matrix $\left[\mu\left( \pm u_{6}+\Sigma_{i \neq 6} a_{i 6} u_{i}, \ldots,-\right)\right]_{\omega}$ is:

$$
\left(\begin{array}{cccccc}
\ddots & & & & & \\
a_{16} & 0 & 0 & 0 & 0 & 0 \\
0 & 0 & 0 & \pm 1+a_{16} & 0 & 0 \\
0 & 0 & \pm 1+a_{16} & 0 & 0 & 0 \\
0 & 0 & 0 & 0 & 0 & \pm 1 \\
0 & 0 & 0 & 0 & \pm 1 & 0
\end{array}\right)
$$


where the other entries may be nonzero. In any case, no matter what $a_{16}$ is, the rank of this matrix will be greater than two. Hence, $\mu$ and $\eta$ cannot be the same trilinear form, and $M \# S^{2} \times\left(S^{2} \times S^{2} \# S^{2} \times S^{2}\right) \# S^{2} \times S^{2} \times S^{2}$.

\section{BIBLIOGRAPHY}

1. G. E. Bredon, Introduction to compact transformation groups, Academic Press, New York, 1972.

2. Marvin Greenberg, Lectures on algebraic topology, Benjamin, New York and Amsterdam, 1967. MR 35 \#6137.

3. A. Haefliger, Differentiable embeddings of $S^{n}$ in $s^{n+q}$ for $q>2$, Ann. of Math. (2) 83 (1966), 402-436. MR 34 \#2024; erratum, 34, p. 1786.

4. - Differentiable links, Topology 1 (1962), 241-244. MR 26 \#5585.

5. Knotted (4k-1)-spheres in 6k-space, Ann. of Math. (2) 75 (1962), 452-

466. MR $26 \# 3070$.

6. J. F. P. Hudson, Piecewise linear topology, Benjamin, New York and Amsterdam, 1969. MR 40 \#2094.

7. Sze-Tsen Hu, Homotopy theory, Pure and Appl. Math., vol. 8, Academic Press, New York and London, 1959. MR 21 \#5186.

8. S. Kim, D. McGavran and J. Pak, Torus group actions on simply connected manifolds, Pacific J. Math. 53 (1974), 435-444. 43 \#6946.

9. Lloyd L. Lininger, $S^{1}$-actions on 6-manifolds, Topology 9 (1971), 301-308. MR

10. William S. Massey, Algebraic topology: An introduction, Harcourt, Brace \& World, New York, 1967. MR 35 \#2271.

11. Dennis McGavran, $T^{3}$ actions on simply connected 6-manifolds, Ph. D. Dissertation, Wayne State University, 1974.

12. J. Milnor and J. Stasheff, Characteristic classes, Ann. of Math. Studies, no. 76, Princeton Univ. Press, Princeton, N. J., 1974.

13. J. Milnor, The representation rings of some classical groups, Mimeographed Notes, Princeton University, Princeton, N. J., 1963.

14. P. Orlik and F. Raymond, Actions of SO(2) on 3-manifolds, Proc. Conf. on Transformation Groups (New Orleans, La., 1967), Springer-Verlag, New York, 1968, pp. 297318. MR 41 \#7717.

15. Actions of the torus on 4-manifolds. I, Trans. Amer. Math.Soc. 152 (1970), 531-559. MR 42 \#3808.

16. J. Pak, Actions of the torus $T^{n}$ on $(n+1)$-manifolds $M^{n+1}$, Pacific J. Math. 44 (1973), 671-674. MR 48 \#1253.

17. F. Raymond, Classification of the actions of the circle on 3-manifolds, Trans. Amer. Math. Soc. 131 (1968), 51-78. MR 36 \#2169.

18. E. H. Spanier, Algebraic topology, McGraw-Hill, New York, 1966. MR 35 \#1007.

19. N. E. Steenrod, The topology of fibre bundles, Princeton Math. Series, vol. 14, Princeton Univ. Press, Princeton, N. J., 1951. MR 12, 522.

20. C. T. C. Wall, Classification problems in differential topology. V. On certain 6manifolds, Invent. Math. 1 (1966), 355-374; corrigendum, ibid. 2 (1967), 306. MR 35 \#6154.

\footnotetext{
DEPARTMENT OF MATHEMATICS, UNIVERSITY OF CONNECTICUT, WATERBURY, CONNECTICUT 06710
} 